Article

\title{
A Multi-Criteria Group Decision-Making Method with Possibility Degree and Power Aggregation Operators of Single Trapezoidal Neutrosophic Numbers
}

\author{
Xiaohui Wu ${ }^{1,2,3}$, Jie Qian ${ }^{1,2, *}$, Juanjuan Peng ${ }^{1,2}$ and Changchun Xue ${ }^{1,2}$ \\ 1 Institute of Big Data Intelligent Decision-Making, Hubei University of Automotive Technology, \\ Shiyan 442002, China; xhw588@huat.edu.cn (X.W.); 20070016@huat.edu.cn (J.P.); \\ xuechangchun@huat.edu.cn (C.X.) \\ 2 School of Economics and Management, Hubei University of Automotive Technology, Shiyan 442002, China \\ 3 School of Business, Central South University, Changsha 410083, China \\ * $\quad$ Correspondence: jieqian@huat.edu.cn; Tel./Fax: +86-719-821-7386
}

Received: 4 October 2018; Accepted: 27 October 2018; Published: 2 November 2018

check for updates

\begin{abstract}
Single valued trapezoidal neutrosophic numbers (SVTNNs) are very useful tools for describing complex information, because of their advantage in describing the information completely, accurately and comprehensively for decision-making problems. In the paper, a method based on SVTNNs is proposed for dealing with multi-criteria group decision-making (MCGDM) problems. Firstly, the new operations SVTNNs are developed for avoiding evaluation information aggregation loss and distortion. Then the possibility degrees and comparison of SVTNNs are proposed from the probability viewpoint for ranking and comparing the single valued trapezoidal neutrosophic information reasonably and accurately. Based on the new operations and possibility degrees of SVTNNs, the single valued trapezoidal neutrosophic power average (SVTNPA) and single valued trapezoidal neutrosophic power geometric (SVTNPG) operators are proposed to aggregate the single valued trapezoidal neutrosophic information. Furthermore, based on the developed aggregation operators, a single valued trapezoidal neutrosophic MCGDM method is developed. Finally, the proposed method is applied to solve the practical problem of the most appropriate green supplier selection and the rank results compared with the previous approach demonstrate the proposed method's effectiveness.
\end{abstract}

Keywords: single valued trapezoidal neutrosophic number; multi-criteria group decision making; possibility degree; power aggregation operators

\section{Introduction}

Multi-criteria decision-making (MCDM) problems are important issues in practice and many MCDM methods have been proposed to deal with such issues. Due to the vagueness of human being thinking and the increased complexity of the objects, there are always much uncertainty, incomplete, indeterminate and inconsistent information in evaluating objects. Traditionally, vagueness information is always described by fuzzy sets (FSs) [1] using the membership function, intuitionistic fuzzy sets (IFSs) [2] using membership and non-membership functions and hesitant fuzzy sets (HFSs) [3] using one/several possible membership degrees. Many fuzzy methods are proposed, for example, Medina [4] extends the fuzzy soft set by Multi-adjoint concept lattices, Pozna \& Precup [5] proposed the operator and application to a fuzzy model, Jane et al. [6] proposed fuzzy S-tree for medical image retrieval and Kumar \& Jarial [7] proposed a hybrid clustering method based on an improved artificial bee 
colony and fuzzy c-means algorithm. However, fuzzy sets cannot deal with the indeterminate information and inconsistent information which exists commonly in complex MCDM problems. As a generalization of the IFSs [2], neutrosophic sets (NSs) [8-10] are proposed to deal with the uncertainty, incomplete, indeterminate and inconsistent information by using the truth-membership, indeterminacy-membership and falsity-membership functions.

Due to the advantages of handling uncertainty, imprecise, incomplete, indeterminate and inconsistent information existing in real world, NSs have attracted many researchers' attentions However NSs are proposed from the philosophical point of view, it is difficult to be directly applied in real scientific and engineering areas without specific descriptions. Therefore, in accordance with the real demand difference, three main subsets of NSs were proposed, namely single valued neutrosophic sets (SVNSs) [11], interval neutrosophic sets (INSs) [12] and multi-valued neutrosophic set (MVNSs) [13]. Based on the aforementioned sets by specifying the NSs, many MCDM methods were developed, which can be classified as three main aspects: aggregation operators, measures and the extension of classic decision-making methods. These methods have been successfully applied in many areas, such as medical diagnosis [14,15], medical treatment [16], neural networks [17], supplier selection [18,19] and green product development [20].

With regard to aggregation operators of SVNSs, Liu and Wang [21] proposed a single-valued neutrosophic normalized weighted Bonferroni mean operator, Liu et al. [22] proposed the generalized neutrosophic operators, Sahin [23] developed the neutrosophic weighted operators. Considering real situations, INSs is more suitable and flexible for describing incomplete information than SVNs. Sun et al. [24] introduced the interval neutrosophic number Choquet integral operator, Ye [25] proposed the interval neutrosophic number ordered weighted operators, Zhang et al. [26] proposed the interval neutrosophic number weighted operators. All of these methods demonstrate the effectiveness.

In respect of measures, Sahin and Kucuk [27] proposed the subset-hood measure for SVNSs, Ye [28-30] and Wu et al. [31] developed some measures of SVNSs including the weighted correlation coefficient [28], cross-entropy [29,31], similarity measure [30]. Broumi and Smarandache proposed the correlation coefficient [32] and cosine similarity measure [33] distance [34] of INSs, Ye [35] proposed the similarity measures between INSs, Sahin and Karabacak [36] developed the inclusion measure for INSs. All of these measures are verified by real cases and demonstrate the effectiveness as well.

In respect of the extension of classic decision-making methods, Zhang and $\mathrm{Wu}$ [19] developed an extended TOPSIS method for the MCDM with incomplete weight information under a single valued neutrosophic environment; Biswas et al. [37] developed the entropy based grey relational analysis method to deal with MCDM problems in which all the criteria weight information described by SVNSs is unknown; Peng et al. [38] developed the outranking approach for MCDM problems based on ELECTRE method; and Sahin and Yigider [39] developed a MCGDM method based on the TOPSIS method for dealing with supplier selection problems. Chi and Liu [40] developed the extended TOPSIS method for deal MCDM problems based on INSs.

Peng et al. [13] firstly defined MVN and developed the approach for solving MCGDM problems based on the multi-valued neutrosophic power weighted operators. Wang and $\mathrm{Li}$ [41] proposed the Hamming distance between multi-valued neutrosophic numbers (MVNN) and the extended TODIM method for dealing with MCDM problems. Wu et al. [42] proposed the novel MCDM methods based on several cross-entropy measures of MVNSs.

However, these subsets of NSs cannot describe the assessment information with different dimensions. For overcoming the shortcomings and improving the flexibility and practicality of these sets, by extending the concept of trapezoidal intuitionistic fuzzy numbers (TrIFNs) [43], single valued trapezoidal neutrosophic numbers (SVTNNs) [44] are proposed for improving the ability to describe complex indeterminate and inconsistent information. Then, SVTNNs attract the attention of some researchers on them as very useful tools on describing evaluation information. Based on SVTNNs, Ye [44] developed the MCDM method on the basis of trapezoidal neutrosophic weighted arithmetic averaging (TNWAA) operator or trapezoidal neutrosophic weighted geometric averaging (TNWGA) 
operator. However, the correlation of trapezoidal numbers and three membership degrees has been ignored and the indeterminate-membership degree is regarded to be equal to falsity-membership degree in these operators, which will lead to information distortion and loss. Meanwhile, it does not take into account the information about the relationships among the assessment information being aggregated, which always exists in the process of solving MCDM problems. To overcome this shortcoming, motivated by the ideal of power aggregation operators [45,46], considering the relationship among the information being aggregated and the possibility degree widely used as a very useful tool to aggregate and rank uncertain data from the probability viewpoint, in this paper we propose the possibility degrees of SVTNNs, single trapezoidal neutrosophic power average (SVTNPA) and single valued trapezoidal neutrosophic power geometric (SVTNPG) operators to deal with MCGDM problems. The prominent characteristics of these proposed operators are taking into account relationship among the aggregation information and overcome the drawbacks of the existing operator of SVTNNs. Then, we utilize these operators and possibility degrees to develop a novel single valued trapezoidal neutrosophic MCGDM method.

The motivation and main attribution of the paper are presented as below:

(1) The novel operation laws of SVTNNs are conducted to overcome the lack of operation laws of SVTNNs appeared in previous paper.

(2) Based on the novel operations of SVTNNs, the SVTNPA and SVTNPG operators are developed.

(3) Based on the concept of the possibility degree, the possibility degree of SVTNNs is defined and presented.

(4) Based on possibility degree of SVTNNs, SVTNPA and SVTNPG operators, a novel method for solving MCGDM problems under single trapezoidal neutrosophic environment is developed.

The rest of the paper is organized as follows. In Section 2, we introduce some basic concepts and operators related to subsets of NS. In Section 3, we propose new operations, possibility degrees and comparison of SVTNNs. SVTNPA and SVTNPG operators are developed in Section 4. The method for solving MCGDM problems under single trapezoidal neutrosophic environment is developed in Section 5. An illustrative example for selecting the most appropriate green supplier for Shanghai General Motors Company is provided in Section 6. Meanwhile a comparison with other method is presented to show the effectiveness of the proposed approach. Finally, conclusions are drawn in Section 7.

\section{Preliminaries}

In this section, some basic concepts, definitions of SVTNNs and two aggregation operators are introduced, which are laying groundwork of latter analysis.

\subsection{NS and SVNS}

Definition 1 ([14]). Let $X$ be a space of points (objects), with a generic element in $X$ denoted by $x$. A NS $A$ in $X$ is characterized by three membership functions, namely truth-membership function $T_{A}(x)$, indeterminacy-membership function $I_{A}(x)$ and falsity-membership function $F_{A}(x)$, where $T_{A}(x), I_{A}(x)$ and $F_{A}(x)$ are real standard or nonstandard subsets of $]^{-} 0,1^{+}\left[\text {, i.e., } T_{A}(x): X \rightarrow\right]^{-} 0,1^{+}\left[, I_{A}(x): X \rightarrow\right]^{-} 0,1^{+}[$ and $\left.F_{A}(x): X \rightarrow\right]^{-} 0,1^{+}$. Therefore, it is no restriction on the sum of $T_{A}(x), I_{A}(x)$ and $F_{A}(x)$ and $-0 \leq T_{A}(x)+I_{A}(x)+F_{A}(x) \leq 3^{+}$.

The neutrosophic set needs to be specified from a technical point of view, otherwise it is difficult to apply in the real scientific and engineering areas. Therefore, Wang et al. [13] proposed the concept SVNS as an instance of neutrosophic set for easily operating and conveniently applying in practical issues. 
Definition 2 ([13]). Let X be a space of points (objects). A SVNS A in X can be expressed as follows:

$$
A=\left\{x,\left\langle T_{A}(x), I_{A}(x), F_{A}(x)\right\rangle \mid x \in X\right\},
$$

where $T_{A}(x) \in[0,1], I_{A}(x) \in[0,1]$ and $F_{A}(x) \in[0,1]$

Obviously, the sum of $T_{A}(x), I_{A}(x)$ and $F_{A}(x)$ satisfies the condition $0 \leq T_{A}(x)+I_{A}(x)+F_{A}(x) \leq 3$.

\subsection{The Trapezoidal Fuzzy Number and SVTNNs}

Definition $3([43,47])$. Let $\widetilde{a}$ be a trapezoidal fuzzy number $\widetilde{a}=\left(a_{1}, a_{2}, a_{3}, a_{4}\right)$ and $a_{1} \leq a_{2} \leq a_{3} \leq a_{4}$. Then its membership function $\mu_{\tilde{a}}(x): R \rightarrow[0,1]$ can be defined as follows:

$$
\mu_{\widetilde{a}}(x)= \begin{cases}\left(x-a_{1}\right) \mu_{\widetilde{a}} /\left(a_{2}-a_{1}\right), & a_{1} \leq x<a_{2} \\ \mu_{\widetilde{a}}, & a_{2} \leq x \leq a_{3} \\ \left(a_{4}-x\right) \mu_{\widetilde{a}} /\left(a_{4}-a_{3}\right), & a_{3}<x \leq a_{4} \\ 0, & \text { otherwise }\end{cases}
$$

Because of the great validity and feasibility of trapezoidal fuzzy numbers and SVNSs in decision-making problems, Ye [44] developed the SVTNNs by combining the two concepts.

Definition 4 ([44]). Let $U$ be a space of points (objects). Then a SVTNN $\alpha$ can be represented as

$$
\alpha=\left\langle\left[a_{1}, a_{2}, a_{3}, a_{4}\right],(T(\alpha), I(\alpha), F(\alpha))\right\rangle
$$

whose truth-membership $T(\alpha)$, indeterminacy-membership $I(\alpha)$ and falsity-membership $F(\alpha)$ can be described as follows:

$$
\begin{gathered}
T(\alpha)= \begin{cases}\left(x-a_{1}\right) T(\alpha) /\left(a_{2}-a_{1}\right), & a_{1} \leq x<a_{2} \\
T(\alpha), & a_{2} \leq x \leq a_{3} \\
\left(a_{4}-x\right) T(\alpha) /\left(a_{4}-a_{3}\right), & a_{3}<x \leq a_{4} \\
0, & \text { otherwise }\end{cases} \\
I(\alpha)= \begin{cases}\left(x-a_{1}\right) I(\alpha) /\left(a_{2}-a_{1}\right), & a_{1} \leq x<a_{2} \\
I(\alpha), & a_{2} \leq x \leq a_{3} \\
\left(a_{4}-x\right) I(\alpha) /\left(a_{4}-a_{3}\right), & a_{3}<x \leq a_{4} \\
0, & \text { otherwise }\end{cases} \\
F(\alpha)= \begin{cases}\left(x-a_{1}\right) F(\alpha) /\left(a_{2}-a_{1}\right), & a_{1} \leq x<a_{2} \\
F(\alpha), & a_{2} \leq x \leq a_{3} \\
\left(a_{4}-x\right) F(\alpha) /\left(a_{4}-a_{3}\right), & a_{3}<x \leq a_{4} \\
0, & \text { otherwise. }\end{cases}
\end{gathered}
$$

Especially, if $a_{1} \geq 0$ and $a_{4}>0$, then $\alpha=\left\langle\left[a_{1}, a_{2}, a_{3}, a_{4}\right],(T(\alpha), I(\alpha), F(\alpha))\right\rangle$ becomes a positive SVTNN. If $I(\alpha)=1-T(\alpha)-F(\alpha)$, then the SVTNN is a TrIFN. And if $I(\alpha)=0, F(\alpha)=0$, then the SVTNN becomes a trapezoidal fuzzy number, that is $\alpha=\left\langle\left[a_{1}, a_{2}, a_{3}, a_{4}\right], T(\alpha)\right\rangle$. 
Example 1. Let $\alpha_{1}=\langle[0.3,0.4,0.7,0.8],(0.8,0.2,0.4)\rangle$ be a SVTNN. Then its truth-membership $T\left(\alpha_{1}\right)$, indeterminacy-membership $I\left(\alpha_{1}\right)$ and falsity-membership $F\left(\alpha_{1}\right)$ can be obtained, respectively, as follows:

$$
\begin{aligned}
& T\left(\alpha_{1}\right)= \begin{cases}8(x-0.3), & 0.3 \leq x<0.4 \\
0.8, & 0.4 \leq x \leq 0.7 \\
8(0.8-x), & 0.7<x \leq 0.8 \\
0, & \text { otherwise. }\end{cases} \\
& I\left(\alpha_{1}\right)= \begin{cases}2(x-0.3), & 0.3 \leq x<0.4 \\
0.2, & 0.4 \leq x \leq 0.7 \\
2(0.8-x), & 0.7<x \leq 0.8 \\
0, & \text { otherwise }\end{cases} \\
& F\left(\alpha_{1}\right)= \begin{cases}4(x-0.3), & 0.3 \leq x<0.4 \\
0.4, & 0.4 \leq x \leq 0.7 \\
4(0.8-x), & 0.7<x \leq 0.8 \\
0, & \text { otherwise. }\end{cases}
\end{aligned}
$$

\section{3. $P A$ and $P G$ Operators}

The power average (PA) operator was firstly proposed by Yager [45]; then, based on PA operator, $\mathrm{Xu}$ and Yager [46] developed the power geometric (PG) operator.

Definition $5([45,46])$. Let $\widetilde{h}=\left\{h_{1}, h_{2}, \cdots, h_{n}\right\}$ a collection of positive real numbers, then PA operator and $P G$ operator can be defined, respectively, as follows:

$$
\begin{gathered}
P A\left(h_{1}, h_{2}, \cdots, h_{n}\right)=\sum_{i=1}^{n} \frac{\left(1+G\left(h_{i}\right)\right) h_{i}}{\sum_{i=1}^{n}\left(1+G\left(h_{i}\right)\right)} \\
P G\left(h_{1}, h_{2}, \cdots, h_{n}\right)=\prod_{i=1}^{n}\left(h_{i}\left(\left(1+G\left(h_{i}\right)\right) / \sum_{i=1}^{n}\left(1+G\left(h_{i}\right)\right)\right)\right)
\end{gathered}
$$

where $G\left(h_{i}\right)=\sum_{j=1, j \neq i}^{n} \operatorname{Sup}\left(h_{i}, h_{j}\right), i=1,2, \cdots, n$. Sup $\left(h_{i}, h_{j}\right)$ is the support for $h_{i}$ from $h_{j}$, satisfying the following properties:

(1) $\operatorname{Sup}\left(h_{i}, h_{j}\right) \in[0,1]$.

(2) $\operatorname{Sup}\left(h_{i}, h_{j}\right)=\operatorname{Sup}\left(h_{j}, h_{i}\right)$.

(3) If $\left|h_{i}-h_{j}\right| \leq|a-b|$, then $\operatorname{Sup}\left(h_{i}, h_{j}\right) \geq \operatorname{Sup}(a, b)$, where $a$ and $b$ are two positive real numbers.

\section{New Operations and Comparison of SVTNNs}

In this section, new operations and comparison method of SVTNNs are proposed for overcoming the limitations in Reference [44] which can avoid information loss and distortion effectively.

\subsection{The New Operations of SVTNNs}

In order to aggregate different SVTNNs in decision-making process, Ye [44] defined the operations of SVTNNs.

Definition 6 ([44]). Let $\alpha=\left\langle\left[a_{1}, a_{2}, a_{3}, a_{4}\right],(T(\alpha), I(\alpha), F(\alpha))\right\rangle$ and $\beta=$ $\left\langle\left[b_{1}, b_{2}, b_{3}, b_{4}\right],(T(\beta), I(\beta), F(\beta))\right\rangle$ be two positive SVTNNs, $0 \leq a_{1} \leq a_{2} \leq a_{3} \leq a_{4} \leq 1$, $0 \leq b_{1} \leq b_{2} \leq b_{3} \leq b_{4} \leq 1, \zeta \geq 0$. Then the operations of SVTNNs can be defined as follows: 
(1) $\alpha+\beta=\left\langle\left[a_{1}+b_{1}, a_{2}+b_{2}, a_{3}+b_{3}, a_{4}+b_{4}\right],(T(\alpha)+T(\beta)-T(\alpha) T(\beta), I(\alpha) I(\beta), F(\alpha) F(\beta))\right\rangle ;$

(2) $\alpha \beta=\left\langle\left[a_{1} b_{1}, a_{2} b_{2}, a_{3} b_{3}, a_{4} b_{4}\right],(T(\alpha) T(\beta), I(\alpha)+I(\beta)-I(\alpha) I(\beta), F(\alpha)+F(\beta)-F(\alpha) F(\beta))\right\rangle$;

(3) $\zeta \alpha=\left\langle\left[\zeta a_{1}, \zeta a_{2}, \zeta a_{3}, \zeta a_{4}\right],\left(1-(1-T(\alpha))^{\zeta},(I(\alpha))^{\zeta},(F(\alpha))^{\zeta}\right)\right\rangle$;

(4) $\alpha^{\zeta}=\left\langle\left[a_{1}^{\zeta}, a_{2}^{\zeta}, a_{3}^{\zeta}, a_{4}^{\zeta}\right],\left((T(\alpha))^{\zeta}, 1-(1-I(\alpha))^{\zeta}, 1-(1-F(\alpha))^{\zeta}\right)\right\rangle$;

However, there are some shortcomings in Definition 7.

(1) The trapezoidal fuzzy numbers and three membership degrees of SVTNNs are considered as two separate parts and operated individually in the operation $\alpha+\beta$, which ignore the correlation among them and cannot reflect the actual results.

Example 2. Let $\alpha_{1}=\langle[0.5,0.6,0.7,0.8],(0,0,1)\rangle$ and $\alpha_{2}=\langle[0.2,0.3,0.4,0.5],(1,0,0)\rangle$ be two SVTNNs.

$$
\alpha_{1}+\alpha_{2}=\langle[0.5,0.6,0.7,0.8],(0,0,1)\rangle+\langle[0.2,0.3,0.4,0.5],(1,0,0)\rangle=\langle[0.7,0.9,1.1,1.3],(1,0,0)\rangle ;
$$

This result is inaccurate since the falsity-membership of $\alpha_{1}$, the correlations among trapezoidal fuzzy numbers and the membership degrees of $\alpha_{1}$ and $\alpha_{2}$ are not considered. Thus, the operations would be unreasonable.

(2) The three membership degrees of SVTNNs are also operated as the trapezoidal fuzzy numbers in the operation $\zeta \alpha$, which can produce the repeat operation and make the result bias.

Example 3. Let $\alpha_{1}=\langle[0.03,0.05,0.07,0.09],(0.3,0.5,0.5)\rangle$ be a $S V T N N, \zeta=10$. Then the result $\zeta \alpha_{1}$ can be obtained by using Definition 6 .

$$
10 \alpha_{1}=\langle[0.3,0.5,0.7,0.9],(0.9718,0.001,0.001)\rangle
$$

The three membership degrees of these SVTNNs are operated repeatedly which make the result distort significantly and conflict with common sense.

For overcoming the limitations existing in the operations proposed by Ye [44], motivated by the operations on triangular intuitionistic fuzzy numbers proposed by Wang et al. [48], new operations of SVTNNs are defined as below.

Definition 7. Let $\alpha=\left\langle\left[a_{1}, a_{2}, a_{3}, a_{4}\right],(T(\alpha), I(\alpha), F(\alpha))\right\rangle$ and $\beta=\left\langle\left[b_{1}, b_{2}, b_{3}, b_{4}\right],(T(\beta), I(\beta), F(\beta))\right\rangle$ be two positive SVTNNs, $0 \leq a_{1} \leq a_{2} \leq a_{3} \leq a_{4} \leq 1,0 \leq b_{1} \leq b_{2} \leq b_{3} \leq b_{4} \leq 1, \zeta \geq 0$. Then the new operations of SVTNNs can be defined as follows:

(1) $\operatorname{neg}(\alpha)=\left\langle\left[1-a_{4}, 1-a_{3}, 1-a_{2}, 1-a_{1}\right],(T(\alpha), I(\alpha), F(\alpha))\right\rangle$;

(2) $\alpha \oplus \beta=\left\langle\left[a_{1}+b_{1}, a_{2}+b_{2}, a_{3}+b_{3}, a_{4}+b_{4}\right],\left(\frac{\varphi(\alpha) T(\alpha)+\varphi(\beta) T(\beta)}{\varphi(\alpha)+\varphi(\beta)}, \frac{\varphi(\alpha) I(\alpha)+\varphi(\beta) I(\beta)}{\varphi(\alpha)+\varphi(\beta)}\right.\right.$, $\left.\left.\frac{\varphi(\alpha) F(\alpha)+\varphi(\beta) F(\beta)}{\varphi(\alpha)+\varphi(\beta)}\right)\right\rangle$, where $\varphi(\alpha)=\frac{a_{1}+2 a_{2}+2 a_{3}+a_{4}}{6}, \varphi(\beta)=\frac{b_{1}+2 b_{2}+2 b_{3}+b_{4}}{6} ;$

(3) $\alpha \otimes \beta=\left\langle\left[a_{1} b_{1}, a_{2} b_{2}, a_{3} b_{3}, a_{4} b_{4}\right],(T(\alpha) T(\beta), I(\alpha)+I(\beta)-I(\alpha) I(\beta), F(\alpha)+F(\beta)-F(\alpha) F(\beta))\right\rangle$;

(4) $\zeta \alpha=\left\langle\left[\zeta a_{1}, \zeta a_{2}, \zeta a_{3}, \zeta a_{4}\right],(T(\alpha), I(\alpha), F(\alpha))\right\rangle$;

(5) $\alpha^{\zeta}=\left\langle\left[a_{1}^{\zeta}, a_{2}^{\zeta}, a_{3}^{\zeta}, a_{4}^{\zeta}\right],\left((T(\alpha))^{\zeta}, 1-(1-I(\alpha))^{\zeta}, 1-(1-F(\alpha))^{\zeta}\right)\right\rangle$;

$\zeta \alpha, \alpha \oplus \beta, \alpha \otimes \beta$ and $\alpha^{\zeta}$ do not appear alone in application due to the meaninglessness of their results. Only in the aggregation process do $\alpha \oplus \beta$ and/or $\alpha \otimes \beta$ being combined with $\zeta \alpha$ and/or $\alpha^{\zeta}$ make sense. 
Example 4. Let $\alpha_{1}=\langle[0.5,0.6,0.7,0.8],(0,0,1)\rangle$ and $\alpha_{2}=\langle[0.2,0.3,0.4,0.5],(1,0,0)\rangle$ be two SVTNNs, $\zeta=2$, the following results can be obtained based on Definition 7 .

(1) $n e g\left(\alpha_{1}\right)=\langle[0.5,0.6,0.8,0.9],(0.4,0.1,0.5)\rangle$;

(2) $\quad \alpha_{1} \oplus \alpha_{2}=\langle[0.3,0.5,1.0,1.2],(0.64,0.22,0.26)\rangle ;$

(3) $\alpha_{1} \otimes \alpha_{2}=\langle[0.02,0.06,0.24,0.35],(0.32,0.37,0.55)\rangle$;

(4) $2 \alpha_{1}=\langle[0.2,0.4,0.8,1.0],(0.4,0.1,0.5)\rangle$;

(5) $\alpha_{1}^{2}=\langle[0.04,0.09,0.25,0.36],(0.16,0.19,0.75)\rangle$.

Compared with the operations proposed by Ye [44], the new operations of SVTNNs have some excellent advantages on reflecting the effect of all truth, indeterminacy and falsity membership degrees of SVTNNs on aggregation results and taking into account the correlation of the trapezoidal fuzzy numbers and three membership degrees of SVTNNs, which can avoid information loss and distortion effectively.

In terms of the corresponding operations of SVTNNs, the following theorem can be easily proved.

Theorem 1. Let $\alpha_{1}, \alpha_{2}, \alpha_{3}$ be three SVTNNs and $\zeta \geq 0$. Then the following equations must be true and easy to proof.

(1) $\alpha_{1} \oplus \alpha_{2}=\alpha_{2} \oplus \alpha_{1}$;

(2) $\left(\alpha_{1} \oplus \alpha_{2}\right) \oplus \alpha_{3}=\alpha_{1} \oplus\left(\alpha_{2} \oplus \alpha_{3}\right)$;

(3) $\alpha_{1} \otimes \alpha_{2}=\alpha_{2} \otimes \alpha_{1}$

(4) $\left(\alpha_{1} \otimes \alpha_{2}\right) \otimes \alpha_{3}=\alpha_{1} \otimes\left(\alpha_{2} \otimes \alpha_{3}\right)$;

(5) $\zeta \alpha_{1} \oplus \zeta \alpha_{2}=\zeta\left(\alpha_{2} \oplus \alpha_{1}\right)$;

(6) $\left(\alpha_{2} \otimes \alpha_{1}\right)^{\tau}=\alpha_{1}^{\tau} \otimes \alpha_{2}^{\tau}$.

\subsection{The Possibility Degree}

The possibility degree, which is proposed from the probability viewpoint, is a very useful tool to rank uncertain data reasonably and accurately.

Definition $8([49,50])$. Let $y=\left[y_{1}, y_{2}\right] \subseteq[0,1]$ and $z=\left[z_{1}, z_{2}\right] \subseteq[0,1]$ be two real number intervals with uniform probability distribution, the probability $y \geq z$ can be represented as $p(y \geq z)$, which exists the following properties:

(1) $0 \leq p(y \geq z) \leq 1$.

(2) $p(y \geq z)+p(z \geq y)=1$.

(3) If $y=z$, then $p(y \geq z)=p(z \geq y)=0.5$.

(4) If $\xi$ is an arbitrary interval or number, $p(y \geq z) \geq 0.5, p(z \geq \xi) \geq 0.5$, then $p(y \geq \xi) \geq 0.5$.

(5) If $\min (y)>\max (z)$, then $p(y \geq z)=1$.

Based on the concept of the possibility degree, the possibility degree of two arbitrary positive SVTNNs is presented. 
Definition 9. Let $\alpha=\left\langle\left[a_{1}, a_{2}, a_{3}, a_{4}\right],(T(\alpha), I(\alpha), F(\alpha))\right\rangle$ and $\beta=\left\langle\left[b_{1}, b_{2}, b_{3}, b_{4}\right],(T(\beta), I(\beta), F(\beta))\right\rangle$ be two positive SVTNNs, $0 \leq a_{1} \leq a_{2} \leq a_{3} \leq a_{4} \leq 1,0 \leq b_{1} \leq b_{2} \leq b_{3} \leq b_{4} \leq 1$. Then the possibility degree of $\alpha \succ \beta p(\alpha \succ \beta)$ can be defined as follows:

$$
\begin{aligned}
p(\alpha \succ \beta)= & \frac{1}{2+\gamma}\left(\max \left\{1-\max \left[\frac{\sum_{i=1}^{4} \max \left(b_{i}-a_{i}, 0\right)+\left(b_{4}-a_{1}\right)+2 \max (T(\beta)-T(\alpha), 0)}{\sum_{i=1}^{4}\left|b_{i}-a_{i}\right|+\left(b_{4}-b_{1}\right)+\left(a_{4}-a_{1}\right)+2|T(\beta)-T(\alpha)|}, 0\right], 0\right\}\right. \\
& +\gamma \max \left\{1-\max \left[\frac{\sum_{i=1}^{4} \max \left(b_{i}-a_{i}, 0\right)+\left(b_{4}-a_{1}\right)+2 \max (I(\beta)-I(\alpha), 0)}{\sum_{i=1}^{4}\left|b_{i}-a_{i}\right|+\left(b_{4}-b_{1}\right)+\left(a_{4}-a_{1}\right)+2|I(\beta)-I(\alpha)|}, 0\right], 0\right\} \\
& \left.+1-\max \left\{1-\max \left[\frac{\sum_{i=1}^{4} \max \left(b_{i}-a_{i}, 0\right)+\left(b_{4}-a_{1}\right)+2 \max (F(\beta)-F(\alpha), 0)}{\sum_{i=1}^{4}\left|b_{i}-a_{i}\right|+\left(b_{4}-b_{1}\right)+\left(a_{4}-a_{1}\right)+2|F(\beta)-F(\alpha)|}, 0\right], 0\right\}\right),
\end{aligned}
$$

where the value of $\gamma \in[0,1]$ is the coefficient that can reflect the attitudes of decision-makers. $\gamma>0.5, \gamma=0.5$ and $\gamma<0.5$ denotes, respectively, the decision-makers' attitude of optimism, compromise and pessimism.

Example 5. Let $\alpha_{1}=\langle[0.3,0.4,0.7,0.8],(0.8,0.2,0.4)\rangle$ and $\alpha_{2}=\langle[0.2,0.5,0.6,0.7],(0.6,0.1,0.3)\rangle$ be two SVTNNs, $\gamma=0.5$. The result of $p\left(\alpha_{1} \succ \alpha_{2}\right)$ can be obtained as follows.

Because

$$
\begin{aligned}
& \frac{\sum_{i=1}^{4} \max \left(b_{i}-a_{i}, 0\right)+\left(b_{4}-a_{1}\right)+2 \max \left(T\left(\alpha_{2}\right)-T\left(\alpha_{1}\right), 0\right)}{\sum_{i=1}^{4}\left|b_{i}-a_{i}\right|+\left(b_{4}-b_{1}\right)+\left(a_{4}-a_{1}\right)+2\left|T\left(\alpha_{2}\right)-T\left(\alpha_{1}\right)\right|} \\
= & \frac{\max (0.2-0.3,0)+\max (0.5-0.4,0)+\max (0.6-0.7,0)+\max (0.7-0.8,0)+(0.7-0.4)+2 \max (0.6-0.8,0)}{|0.2-0.3|+|0.5-0.4|+|0.6-0.7|+|0.7-0.8|+(0.7-0.2)+(0.8-0.3)+2|0.6-0.8|} \\
= & \frac{0.4}{1.8}=0.222 ; \\
& \frac{\sum_{i=1}^{4} \max \left(b_{i}-a_{i}, 0\right)+\left(b_{4}-a_{1}\right)+2 \max \left(I\left(\alpha_{2}\right)-I\left(\alpha_{1}\right), 0\right)}{\sum_{i=1}^{4}\left|b_{i}-a_{i}\right|+\left(b_{4}-b_{1}\right)+\left(a_{4}-a_{1}\right)+2\left|I\left(\alpha_{2}\right)-I\left(\alpha_{1}\right)\right|}=\frac{0.4}{1.6}=0.25 ; \\
& \frac{\sum_{i=1}^{4} \max \left(b_{i}-a_{i}, 0\right)+\left(b_{4}-a_{1}\right)+2 \max \left(F\left(\alpha_{2}\right)-F\left(\alpha_{1}\right), 0\right)}{\sum_{i=1}^{4}\left|b_{i}-a_{i}\right|+\left(b_{4}-b_{1}\right)+\left(a_{4}-a_{1}\right)+2\left|F\left(\alpha_{2}\right)-F\left(\alpha_{1}\right)\right|}=\frac{0.4}{1.6}=0.25
\end{aligned}
$$

Therefore, we can obtain

$$
\begin{aligned}
p\left(\alpha_{1} \succ \alpha_{2}\right) & =\frac{1}{2.5}(\max \{1-\max [0.222,0], 0\}+0.5 \times \max \{1-\max [0.25,0], 0\}+1-\max \{1-\max [0.25,0], 0\}) \\
& =\frac{1}{2.5}(0.778+0.5 \times 0.75+0.25) \\
& =0.561
\end{aligned}
$$

Theorem 2. Let $\alpha=\left\langle\left[a_{1}, a_{2}, a_{3}, a_{4}\right],(T(\alpha), I(\alpha), F(\alpha))\right\rangle$ and $\beta=\left\langle\left[b_{1}, b_{2}, b_{3}, b_{4}\right],(T(\beta), I(\beta), F(\beta))\right\rangle$ be two positive SVTNNs, $0 \leq a_{1} \leq a_{2} \leq a_{3} \leq a_{4} \leq 1,0 \leq b_{1} \leq b_{2} \leq b_{3} \leq b_{4} \leq 1$. Then the following properties must be true.

(1) $0 \leq p(\alpha \succ \beta) \leq 1$.

(2) $\quad p(\alpha \succ \beta)+p(\beta \succ \alpha)=1$.

(3) If $a_{i}=b_{i}, i=1,2,3,4, T(\alpha)=T(\beta), I(\alpha)=I(\beta)$ and $F(\alpha)=F(\beta)$, then $p(\alpha \succ \beta)=p(\beta \succ \alpha)=0.5$.

(4) If $\xi$ is an arbitrary positive SVTNN, $p(\alpha \succ \beta) \geq 0.5, p(\beta \succ \xi) \geq 0.5$, then $p(\alpha \geq \xi) \geq 0.5$.

(5) If $a_{1} \geq b_{4}, T(\alpha) \geq T(\beta), I(\alpha) \geq I(\beta)$ and $F(\alpha) \leq F(\beta)$, then $p(\alpha \succ \beta)=1$.

Now we prove the property (2), the proofs of other properties are similar to the proof the property (2), thus, they are omitted.

$$
\begin{aligned}
& \text { Proof. Let } x(\alpha, \beta)=\frac{\sum_{i=1}^{4} \max \left(b_{i}-a_{i}, 0\right)+\left(b_{4}-a_{1}\right)+2 \max (T(\beta)-T(\alpha), 0)}{\sum_{i=1}^{4}\left|b_{i}-a_{i}\right|+\left(b_{4}-b_{1}\right)+\left(a_{4}-a_{1}\right)+2|T(\beta)-T(\alpha)|}, y(\alpha, \beta) \\
& \frac{\sum_{i=1}^{4} \max \left(b_{i}-a_{i}, 0\right)+\left(b_{4}-a_{1}\right)+2 \max (I(\beta)-I(\alpha), 0)}{\sum_{i=1}^{4}\left|b_{i}-a_{i}\right|+\left(b_{4}-b_{1}\right)+\left(a_{4}-a_{1}\right)+2|I(\beta)-I(\alpha)|}, z(\alpha, \beta)=\frac{\sum_{i=1}^{4} \max \left(b_{i}-a_{i}, 0\right)+\left(b_{4}-a_{1}\right)+2 \max (F(\beta)-F(\alpha), 0)}{\sum_{i=1}^{4}\left|b_{i}-a_{i}\right|+\left(b_{4}-b_{1}\right)+\left(a_{4}-a_{1}\right)+2|F(\beta)-F(\alpha)|} .
\end{aligned}
$$


Then

$$
p(\alpha \succ \beta)=\frac{1}{3}(\max \{1-\max [x(\alpha, \beta), 0], 0\}+\gamma \max \{1-\max [y(\alpha, \beta), 0], 0\}+1-\max \{1-\max [z(\alpha, \beta), 0], 0\}) .
$$

Because

$$
\begin{aligned}
& x(\alpha, \beta)+x(\beta, \alpha) \\
& =\frac{\sum_{i=1}^{4} \max \left(b_{i}-a_{i}, 0\right)+\left(b_{4}-a_{1}\right)+2 \max (T(\beta)-T(\alpha), 0)}{\sum_{i=1}^{4}\left|b_{i}-a_{i}\right|+\left(b_{4}-b_{1}\right)+\left(a_{4}-a_{1}\right)+2|T(\beta)-T(\alpha)|}+\frac{\sum_{i=1}^{4} \max \left(a_{i}-b_{i}, 0\right)+\left(a_{4}-b_{1}\right)+2 \max (T(\alpha)-T(\beta), 0)}{\sum_{i=1}^{4}\left|a_{i}-b_{i}\right|+\left(a_{4}-a_{1}\right)+\left(b_{4}-b_{1}\right)+2|T(\alpha)-T(\beta)|} \\
& =\frac{\sum_{i=1}^{4}\left|a_{i}-b_{i}\right|+\left(a_{4}-b_{1}\right)+\left(b_{4}-a_{1}\right)+2|T(\alpha)-T(\beta)|}{\sum_{i=1}^{4}\left|a_{i}-b_{i}\right|+\left(a_{4}-a_{1}\right)+\left(b_{4}-b_{1}\right)+2|T(\alpha)-T(\beta)|}=1 ; \\
& \quad y(\alpha, \beta)+y(\beta, \alpha)=1 ; z(\alpha, \beta)+z(\beta, \alpha)=1 .
\end{aligned}
$$

We can obtain $\max \{1-\max [x(\alpha, \beta), 0], 0\}+\max \{1-\max [x(\beta, \alpha), 0], 0\}=1$; $\max \{1-\max [y(\alpha, \beta), 0], 0\}+\max \{1-\max [y(\beta, \alpha), 0], 0\}=1 ; 1-\max \{1-\max [z(\alpha, \beta), 0], 0\}+$ $1-\max \{1-\max [z(\beta, \alpha), 0], 0\}=1$.

Therefore,

$$
\begin{aligned}
& p(\alpha \succ \beta)+P(\beta \succ \alpha) \\
& =\frac{1}{2+\lambda}(\max \{1-\max [x(\alpha, \beta), 0], 0\}+\lambda \max \{1-\max [y(\alpha, \beta), 0], 0\}+1-\max \{1-\max [z(\alpha, \beta), 0], 0\}) \\
& \quad+\frac{1}{2+\lambda}(\max \{1-\max [x(\beta, \alpha), 0], 0\}+\lambda \max \{1-\max [y(\beta, \alpha), 0], 0\}+1-\max \{1-\max [z(\beta, \alpha), 0], 0\}) \\
& =\frac{1}{2+\lambda}(1+\lambda+1)=1 .
\end{aligned}
$$

The proof of the property (2) is completed now.

\subsection{The Comparison Method of SVTNNs}

In this subsection, based on the concept of the possibility degree of two arbitrary positive SVTNNs defined in Definition 9, the new comparison method for two SVTNNs is presented.

For comparing different SVTNNs in decision-making process, Ye [44] defined the score function and comparison of SVTNNs.

Definition 10 [44]. Let $\alpha=\left\langle\left[a_{1}, a_{2}, a_{3}, a_{4}\right],(T(\alpha), I(\alpha), F(\alpha))\right\rangle$ and $\beta=$ $\left\langle\left[b_{1}, b_{2}, b_{3}, b_{4}\right],(T(\beta), I(\beta), F(\beta))\right\rangle$ be two SVTNNs. Then the score degree of $\alpha S(\alpha)$ can be defined as follows:

$$
S(\alpha)=\frac{1}{12}\left(a_{1}+a_{2}+a_{3}+a_{4}\right) \times(2+T(\alpha)-I(\alpha)-F(\alpha)) .
$$

If $S(\alpha)>S(\beta)$, then $\alpha \succ \beta$; if $S(\alpha)<S(\beta)$, then $\alpha \prec \beta$; if $S(\alpha)=S(\beta)$, then $\alpha \sim \beta$.

However, the score function is operated by assuming that the parameters of trapezoidal fuzzy numbers own same weight, which cannot reflect the different importance for the four parameters of a trapezoidal fuzzy number and make aggregating result bias.

Example 6. Let $\alpha_{1}=\langle[0.1,0.3,0.5,0.6],(0.6,0,0.4)\rangle$ and $\alpha_{2}=\langle[0,0.4,0.5,0.6],(0.6,0,0.4)\rangle$ be two SVTNNs.

$$
S\left(\alpha_{1}\right)=\frac{1}{12}(0.1+0.3+0.5+0.6) \times(2+0.6-0-0.4)=0.275 ; S\left(\alpha_{2}\right)=0.275 .
$$

We cannot compare these two SVTNNs using the above function but it is easy to know that $\alpha_{1}$ is superior to $\alpha_{2}$.

Meanwhile, the function operates the indeterminacy-membership degree as like the false-membership degree, which does not take the preference of decision-makers into consideration. 
Example 7. Let $\alpha_{1}=\langle[0.2,0.3,0.4,0.5],(0.6,0,0.4)\rangle$ and $\alpha_{2}=\langle[0.2,0.3,0.4,0.5],(0.6,0.4,0)\rangle$ be two SVTNNs.

$$
S\left(\alpha_{1}\right)=\frac{1}{12}(0.2+0.3+0.4+0.5) \times(2+0.6-0-0.4)=0.257 ; S\left(\alpha_{2}\right)=0.257 .
$$

$S\left(\alpha_{1}\right)=S\left(\alpha_{2}\right)$ indicates that $\alpha_{1}$ is equal to $\alpha_{2}$. However, it is obvious that $\alpha_{2}$ is superior to $\alpha_{1}$.

These shortcomings existing in the score function given in Definition 10 may make the comparison results of SVTNNs unacceptable. For overcoming the limitations of Definition 10, based on the concept of the possibility degree of two arbitrary positive SVTNNs defined in Definition 9, we propose a new comparison method.

Definition 11. Let $\alpha$ and $\beta$ be two positive SVTNNs, $\gamma$ be an arbitrary positive SVTNN and then the comparison method can be defined as follows.

(1) If $p(\alpha \succ \gamma)>p(\beta \succ \gamma)$, then $\alpha \succ \beta$, i.e., $\alpha$ is superior to $\beta$.

(2) If $p(\alpha \succ \gamma)=p(\beta \succ \gamma)$, then $\alpha \sim \beta$, i.e., $\alpha$ is equal to $\beta$.

(3) If $p(\alpha \succ \gamma)<p(\beta \succ \gamma)$, then $\alpha \prec \beta$, i.e., $\beta$ is superior to $\alpha$.

Example 8. Let $\lambda=0.5$. When using the data of Example 4 and the following can be obtained.

$$
p\left(\alpha_{1} \succ \alpha_{2}\right)=0.508 ; p\left(\alpha_{2} \succ \alpha_{1}\right)=0.492 \text {, so } \alpha_{1} \succ \alpha_{2} \text {. }
$$

When using the data of Example 5 and the following can be obtained.

$$
p\left(\alpha_{1} \succ \alpha_{2}\right)=0.329 ; p\left(\alpha_{2} \succ \alpha_{1}\right)=0.671 \text {, so } \alpha_{2} \succ \alpha_{1} .
$$

Thus, the results of the above two examples are consistent with our common sense. Because the score function can overcome the shortcoming existing in Reference [44] by calculating the indeterminacy-membership degree by taking into account the preference of decision-makers, the results are more grounded in reality than the results obtained by using the score degree proposed by Ye [44].

\section{Single Valued Trapezoidal Neutrosophic Power Aggregation Operators}

In this section, the SVTNPA and SVTNPG operators based on the new operations of SVTNNs are developed.

Definition 12. Let $\alpha_{i}=\left\langle\left[a_{i 1}, a_{i 2}, a_{i 3}, a_{i 4}\right],\left(T\left(\alpha_{i}\right), I\left(\alpha_{i}\right), F\left(\alpha_{i}\right)\right)\right\rangle$ be a collection of positive SVTNNs. Then the single valued trapezoidal neutrosophic power average (SVTNPA) operator can be defined as follows:

$$
\begin{aligned}
\operatorname{SVTNPA}\left(\alpha_{1}, \alpha_{2}, \cdots, \alpha_{n}\right) & =\frac{1+G\left(\alpha_{1}\right)}{\sum_{i=1}^{n}\left(1+G\left(\alpha_{i}\right)\right)} \alpha_{1} \oplus \frac{1+G\left(\alpha_{2}\right)}{\sum_{i=1}^{n}\left(1+G\left(\alpha_{i}\right)\right)} \alpha_{2} \oplus \cdots \oplus \frac{1+G\left(\alpha_{n}\right)}{\sum_{i=1}^{n}\left(1+G\left(\alpha_{i}\right)\right)} \alpha_{n} \\
& =\oplus_{i=1}^{n}\left(\frac{1+G\left(\alpha_{i}\right)}{\sum_{i=1}^{n}\left(1+G\left(\alpha_{i}\right)\right)} \alpha_{i}\right),
\end{aligned}
$$

where $G\left(\alpha_{i}\right)=\sum_{j=1, j \neq i}^{n} \operatorname{Sup}\left(\alpha_{i}, \alpha_{j}\right)$, Sup $\left(\alpha_{i}, \alpha_{j}\right)$ is the support for $\alpha_{i}$ from $\alpha_{j}$, satisfying the following properties.

(1) $\operatorname{Sup}\left(\alpha_{i}, \alpha_{j}\right) \in[0,1]$.

(2) $\operatorname{Sup}\left(\alpha_{i}, \alpha_{j}\right)=\operatorname{Sup}\left(\alpha_{j}, \alpha_{i}\right)$.

(3) If $\left|p\left(\alpha_{i} \succ \alpha_{j}\right)-p\left(\alpha_{j} \succ \alpha_{i}\right)\right|<|p(\pi \succ v)-p(v \succ \pi)|$, then $\operatorname{Sup}\left(\alpha_{i}, \alpha_{j}\right)>\operatorname{Sup}(\pi, v)$, where $\pi$ and $v$ are two positive SVTNNs, $p\left(\alpha_{i} \succ \alpha_{j}\right), p\left(\alpha_{j} \succ \alpha_{i}\right), p(\pi \succ v)$ and $p(v \succ \pi)$ are the possibility degree of $\alpha_{i} \succ \alpha_{j}, \alpha_{j} \succ \alpha_{i}, \pi \succ v$ and $v \succ \pi$. 
The support for $\alpha_{i}$ from $\alpha_{j}$ can be obtained using the function $\operatorname{Sup}\left(\alpha_{i}, \alpha_{j}\right)=1-$ $\left|p\left(\alpha_{i} \succ \alpha_{j}\right)-p\left(\alpha_{j} \succ \alpha_{i}\right)\right|$. Obviously, the closer the values of the score of $\alpha_{i}$ and $\alpha_{j}$, the more they support each other.

Theorem 3. Let $\alpha_{i}=\left\langle\left[a_{i 1}, a_{i 2}, a_{i 3}, a_{i 4}\right],\left(T\left(\alpha_{i}\right), I\left(\alpha_{i}\right), F\left(\alpha_{i}\right)\right)\right\rangle(i=1,2, \cdots, n)$ be a collection of positive SVTNNs. The aggregated result, obtained by using the SVTNPA operator, is also a positive SVTNN, and

$$
\begin{aligned}
\operatorname{SVTNPA}\left(\alpha_{1}, \alpha_{2}, \cdots, \alpha_{n}\right)= & \oplus_{i=1}^{n}\left(\frac{1+G\left(\alpha_{i}\right)}{\sum_{i=1}^{n}\left(1+G\left(\alpha_{i}\right)\right)} \alpha_{i}\right) \\
= & \left\langle\left(\left[\sum_{i=1}^{n}\left(\tau\left(\alpha_{i}\right) a_{i 1}\right), \sum_{i=1}^{n}\left(\tau\left(\alpha_{i}\right) a_{i 2}\right), \sum_{i=1}^{n}\left(\tau\left(\alpha_{i}\right) a_{i 3}\right), \sum_{i=1}^{n}\left(\tau\left(\alpha_{i}\right) a_{i 4}\right)\right],\right.\right. \\
& \left.\left(\frac{\sum_{i=1}^{n}\left(\varphi\left(\alpha_{i}\right) T\left(\alpha_{i}\right)\right)}{\sum_{i=1}^{n} \varphi\left(\alpha_{i}\right)}, \frac{\sum_{i=1}^{n}\left(\varphi\left(\alpha_{i}\right) I\left(\alpha_{i}\right)\right)}{\sum_{i=1}^{n} \varphi\left(\alpha_{i}\right)}, \frac{\sum_{i=1}^{n}\left(\varphi\left(\alpha_{i}\right) F\left(\alpha_{i}\right)\right)}{\sum_{i=1}^{n} \varphi\left(\alpha_{i}\right)}\right)\right\rangle,
\end{aligned}
$$

where $G\left(\alpha_{i}\right)=\sum_{j=1, j \neq i}^{n} \operatorname{Sup}\left(\alpha_{i}, \alpha_{j}\right)$, Sup $\left(\alpha_{i}, \alpha_{j}\right)=1-\left|p\left(\alpha_{i} \succ \alpha_{j}\right)-p\left(\alpha_{j} \succ \alpha_{i}\right)\right|$ is the support for $\alpha_{i}$ from $\alpha_{j}, \tau\left(\alpha_{i}\right)=\frac{1+G\left(\alpha_{i}\right)}{\sum_{i=1}^{n}\left(1+G\left(\alpha_{i}\right)\right)}, \varphi\left(\alpha_{i}\right)=\frac{1}{6}\left(\tau\left(\alpha_{i}\right) a_{i 1}+2 \tau\left(\alpha_{i}\right) a_{i 2}+2 \tau\left(\alpha_{i}\right) a_{i 3}+\tau\left(\alpha_{i}\right) a_{i 4}\right), p\left(\alpha_{i} \succ \alpha_{j}\right)$ and $p\left(\alpha_{j} \succ \alpha_{i}\right)$ are the score functions of $\alpha_{i} \succ \alpha_{j}, \alpha_{j} \succ \alpha_{i}$.

Proof. According to Definition 8, the aggregated result is also a positive SVTNN. Therefore, Theorem 3 can be easily proven by using a mathematical induction on $n$.

(1) For $n=2$, since

$$
\begin{aligned}
& \frac{1+G\left(\alpha_{1}\right)_{1}}{\sum_{i=1}^{2}\left(1+G\left(\alpha_{i}\right)\right)} \alpha_{1}=\left\langle\left[\tau\left(\alpha_{1}\right) a_{11}, \tau\left(\alpha_{1}\right) a_{12}, \tau\left(\alpha_{1}\right) a_{13}, \tau\left(\alpha_{1}\right) a_{14}\right],\left(T\left(\alpha_{1}\right), I\left(\alpha_{1}\right), F\left(\alpha_{1}\right)\right)\right\rangle ; \\
& \frac{1+G\left(\alpha_{2}\right)}{\sum_{i=1}^{2}\left(1+G\left(\alpha_{i}\right)\right)} \alpha_{2}=\left\langle\left[\tau\left(\alpha_{2}\right) a_{21}, \tau\left(\alpha_{2}\right) a_{22}, \tau\left(\alpha_{2}\right) a_{23}, \tau\left(\alpha_{2}\right) a_{24}\right],\left(T\left(\alpha_{2}\right), I\left(\alpha_{2}\right), F\left(\alpha_{2}\right)\right)\right\rangle .
\end{aligned}
$$

Then

$$
\begin{aligned}
& \text { SVTNPA }\left(\alpha_{1}, \alpha_{2}\right)=\frac{1+G\left(\alpha_{1}\right)}{\sum_{i=1}^{2}\left(1+G\left(\alpha_{i}\right)\right)} \alpha_{1} \oplus \frac{1+G\left(\alpha_{2}\right)}{\sum_{i=1}^{2}\left(1+G\left(\alpha_{i}\right)\right)} \alpha_{2} \\
& =\left\langle\left(\left[\tau\left(\alpha_{1}\right) a_{11}+\tau\left(\alpha_{2}\right) a_{21}, \tau\left(\alpha_{1}\right) a_{12}+\tau\left(\alpha_{2}\right) a_{22}, \tau\left(\alpha_{1}\right) a_{13}+\tau\left(\alpha_{2}\right) a_{23}, \tau\left(\alpha_{1}\right) a_{14}+\tau\left(\alpha_{2}\right) a_{24}\right],\right.\right. \\
& \left.\left(\frac{\varphi\left(\alpha_{1}\right) T\left(\alpha_{1}\right)+\varphi\left(\alpha_{2}\right) T\left(\alpha_{2}\right)}{\varphi\left(\alpha_{1}\right)+\varphi\left(\alpha_{2}\right)}, \frac{\varphi\left(\alpha_{1}\right) I\left(\alpha_{1}\right)+\varphi\left(\alpha_{2}\right) I\left(\alpha_{2}\right)}{\varphi\left(\alpha_{1}\right)+\varphi\left(\alpha_{2}\right)}, \frac{\varphi\left(\alpha_{1}\right) I\left(\alpha_{1}\right)+\varphi\left(\alpha_{2}\right) I\left(\alpha_{2}\right)}{\varphi\left(\alpha_{1}\right)+\varphi\left(\alpha_{2}\right)}\right)\right\rangle .
\end{aligned}
$$

(2) If we hold $n=k$, then

$$
\begin{aligned}
\operatorname{SVTNPA}\left(\alpha_{1}, \alpha_{2}, \cdots, \alpha_{n}\right)= & \oplus_{i=1}^{k}\left(\frac{1+G\left(\alpha_{i}\right)}{\sum_{i=1}^{k}\left(1+G\left(\alpha_{i}\right)\right)} \alpha_{i}\right) \\
= & \left\langle\left(\left[\sum_{i=1}^{k}\left(\tau\left(\alpha_{i}\right) a_{i 1}\right), \sum_{i=1}^{k}\left(\tau\left(\alpha_{i}\right) a_{i 2}\right), \sum_{i=1}^{k}\left(\tau\left(\alpha_{i}\right) a_{i 3}\right), \sum_{i=1}^{k}\left(\tau\left(\alpha_{i}\right) a_{i 4}\right)\right],\right.\right. \\
& \left.\left(\frac{\sum_{i=1}^{k}\left(\varphi\left(\alpha_{i}\right) T\left(\alpha_{i}\right)\right)}{\sum_{i=1}^{k} \varphi\left(\alpha_{i}\right)}, \frac{\sum_{i=1}^{k}\left(\varphi\left(\alpha_{i}\right) I\left(\alpha_{i}\right)\right)}{\sum_{i=1}^{k} \varphi\left(\alpha_{i}\right)}, \frac{\sum_{i=1}^{k}\left(\varphi\left(\alpha_{i}\right) F\left(\alpha_{i}\right)\right)}{\sum_{i=1}^{k} \varphi\left(\alpha_{i}\right)}\right)\right\rangle .
\end{aligned}
$$

When $n=k+1$, by the operations described in Definition 10, we have

$$
\begin{aligned}
\operatorname{SVTNPA}\left(\alpha_{1}, \alpha_{2}, \cdots, \alpha_{n}\right)= & \oplus_{i=1}^{k}\left(\frac{1+G\left(\alpha_{i}\right)}{\sum_{i=1}^{n}\left(1+G\left(\alpha_{i}\right)\right)} \alpha_{i}\right) \oplus \frac{1+G\left(\alpha_{k+1}\right)}{\sum_{i=1}^{n}\left(1+G\left(\alpha_{i}\right)\right)} \alpha_{k+1} \\
= & \left\langle\left(\left[\sum_{i=1}^{k+1}\left(\tau\left(\alpha_{i}\right) a_{i 1}\right), \sum_{i=1}^{k+1}\left(\tau\left(\alpha_{i}\right) a_{i 2}\right), \sum_{i=1}^{k+1}\left(\tau\left(\alpha_{i}\right) a_{i 3}\right), \sum_{i=1}^{k+1}\left(\tau\left(\alpha_{i}\right) a_{i 4}\right)\right],\right.\right. \\
& \left.\left(\frac{\sum_{i=1}^{k+1}\left(\varphi\left(\alpha_{i}\right) T\left(\alpha_{i}\right)\right)}{\sum_{i=1}^{k+1} \varphi\left(\alpha_{i}\right)}, \frac{\sum_{i=1}^{k+1}\left(\varphi\left(\alpha_{i}\right) I\left(\alpha_{i}\right)\right)}{\sum_{i=1}^{k+1} \varphi\left(\alpha_{i}\right)}, \frac{\sum_{i=1}^{k+1}\left(\varphi\left(\alpha_{i}\right) F\left(\alpha_{i}\right)\right)}{\sum_{i=1}^{k+1} \varphi\left(\alpha_{i}\right)}\right)\right\rangle .
\end{aligned}
$$

So, $n=k+1$, Theorem 2 is also right.

According to (1) and (2), we can get Theorem 3 hold for any $n$. 
Example 9. Let $\alpha_{1}=\langle[0.3,0.4,0.7,0.8],(0.8,0.2,0.4)\rangle, \alpha_{2}=\langle[0.2,0.5,0.6,0.7],(0.6,0.1,0.3)\rangle$, $\alpha_{3}=\langle[0.3,0.4,0.5,0.6],(0.7,0.3,0.3)\rangle$ and $\alpha_{4}=\langle[0.3,0.5,0.5,0.7],(0.6,0.2,0.3)\rangle$ be four positive SVTNNs, $\lambda=0.8$. Then SVTNPA $\left(\alpha_{1}, \alpha_{2}, \alpha_{3}, \alpha_{4}\right)$ can be calculated as follows.

Because $p\left(\alpha_{1} \succ \alpha_{2}\right)=0.566, p\left(\alpha_{1} \succ \alpha_{3}\right)=0.541, p\left(\alpha_{1} \succ \alpha_{4}\right)=0.547, p\left(\alpha_{2} \succ \alpha_{3}\right)=0.452$, $p\left(\alpha_{2} \succ \alpha_{4}\right)=0.467, p\left(\alpha_{3} \succ \alpha_{4}\right)=0.530$, we can obtain the following results.

$$
\begin{gathered}
G\left(\alpha_{1}\right)=\sum_{j=1, j \neq i}^{3} \operatorname{Sup}\left(\alpha_{1}, \alpha_{j}\right) \\
=\sum_{j=2}^{4}\left(1-\left|p\left(\alpha_{1} \succ \alpha_{j}\right)-p\left(\alpha_{j} \succ \alpha_{1}\right)\right|\right) \\
=(1-|0.566-0.434|)+(1-|0.541-0.459|)+(1-|0.547-0.453|) \\
=2.692, \\
\quad G\left(\alpha_{2}\right)=2.707, G\left(\alpha_{3}\right)=2.762, G\left(\alpha_{4}\right)=2.779 . \\
\begin{array}{c}
\tau\left(\alpha_{1}\right)=\frac{1+G\left(\alpha_{1}\right)}{\sum_{i=1}^{4}\left(1+G\left(\alpha_{i}\right)\right)}=\frac{1+2.692}{(1+2.692)+(1+2.707)+(1+2.762)+(1+2.779)}=0.247, \\
\tau\left(\alpha_{2}\right)=0.248, \tau\left(\alpha_{3}\right)=0.252, \tau\left(\alpha_{4}\right)=0.253 . \\
\varphi\left(\alpha_{1}\right)=\frac{1}{6}\left(\tau\left(\alpha_{1}\right) a_{11}+2 \tau\left(\alpha_{1}\right) a_{12}+2 \tau\left(\alpha_{1}\right) a_{13}+\tau\left(\alpha_{1}\right) a_{14}\right)=\frac{1}{6} \times 0.247 \times(0.3+2 \times 0.4+2 \times 0.7+0.8)=0.136, \\
\varphi\left(\alpha_{2}\right)=0.128, \varphi\left(\alpha_{3}\right)=0.113, \varphi\left(\alpha_{4}\right)=0.127 .
\end{array}
\end{gathered}
$$

Therefore, $\operatorname{SVTNPA}\left(\alpha_{1}, \alpha_{2}, \alpha_{3}, \alpha_{4}\right)=\langle[0.275,0.450,0.574,0.699],(0.676,0.197,0.327)\rangle$.

Theorem 4. Let $\alpha_{i}=\left\langle\left[a_{i 1}, a_{i 2}, a_{i 3}, a_{i 4}\right],\left(T\left(\alpha_{i}\right), I\left(\alpha_{i}\right), F\left(\alpha_{i}\right)\right)\right\rangle(i=1,2, \cdots, n)$ be a collection of positive SVTNNs. If $\operatorname{Sup}\left(\alpha_{i}, \alpha_{j}\right)=c(c \in[0,1], i \neq j, j=1,2, \cdots, n)$, then SVTNPA operator reduces to single valued trapezoidal neutrosophic average (SVTNA) operator as follows:

$$
\operatorname{SVTNPA}\left(\alpha_{1}, \alpha_{2}, \cdots, \alpha_{n}\right)=\operatorname{SVTNA}\left(\alpha_{1}, \alpha_{2}, \cdots, \alpha_{n}\right)=\oplus_{i=1}^{n}\left(\frac{1}{n} \alpha_{i}\right)
$$

Proof. Because $\operatorname{Sup}\left(\alpha_{i}, \alpha_{j}\right)=c(c \in[0,1], i \neq j, j=1,2, \cdots, n)$, we have $G\left(\alpha_{i}\right)=\sum_{j=1, j \neq i}^{n} \operatorname{Sup}\left(\alpha_{i}, \alpha_{j}\right)=$ $(n-1) c$.

Therefore,

$$
\operatorname{SVTNPA}\left(\alpha_{1}, \alpha_{2}, \cdots, \alpha_{n}\right)=\oplus_{i=1}^{n}\left(\frac{1+G\left(\alpha_{i}\right)_{i}}{\sum_{i=1}^{n}\left(1+G\left(\alpha_{i}\right)\right)} \alpha_{i}\right)=\oplus_{i=1}^{n}\left(\frac{1+(n-1) c}{\sum_{i=1}^{n}(1+(n-1) c)} \alpha_{i}\right)=\oplus_{i=1}^{n}\left(\frac{1}{n} \alpha_{i}\right) .
$$

Finally, we can get $\operatorname{SVTNPA}\left(\alpha_{1}, \alpha_{2}, \cdots, \alpha_{n}\right)=\operatorname{SVTN} A\left(\alpha_{1}, \alpha_{2}, \cdots, \alpha_{n}\right)=\oplus_{i=1}^{n}\left(\alpha_{i}\right)$ and the proof of Theorem 4 is completed now.

Definition 13. Let $\alpha_{i}=\left\langle\left[a_{i 1}, a_{i 2}, a_{i 3}, a_{i 4}\right],\left(T\left(\alpha_{i}\right), I\left(\alpha_{i}\right), F\left(\alpha_{i}\right)\right)\right\rangle(i=1,2, \cdots, n)$ be a collection of positive SVTNNs. Then the single valued trapezoidal neutrosophic power geometric (SVTNPG) operator can be defined as follows:

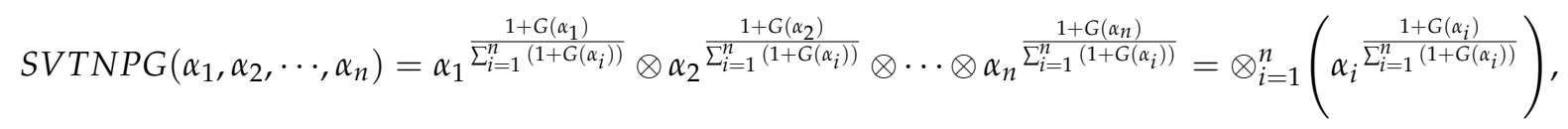

where $G\left(\alpha_{i}\right)=\sum_{j=1, j \neq i}^{n} \operatorname{Sup}\left(\alpha_{i}, \alpha_{j}\right), \operatorname{Sup}\left(\alpha_{i}, \alpha_{j}\right)$ is the support for $\alpha_{i}$ from $\alpha_{j}$. 
Theorem 5. Let $\alpha_{i}=\left\langle\left[a_{i 1}, a_{i 2}, a_{i 3}, a_{i 4}\right],\left(T\left(\alpha_{i}\right), I\left(\alpha_{i}\right), F\left(\alpha_{i}\right)\right)\right\rangle(i=1,2, \cdots, n)$ be a collection of positive SVTNNs. The aggregated result, obtained by using the SVTNPG operator, is also a positive SVTNN, and

$$
\begin{aligned}
& \operatorname{SVTNPG}\left(\alpha_{1}, \alpha_{2}, \cdots, \alpha_{n}\right)=\otimes_{i=1}^{n}\left(\alpha_{i}^{\tau \tau\left(\alpha_{i}\right)}\right) \\
& =\left\langle\left[\prod_{i=1}^{n} a_{i 1}^{\tau\left(\alpha_{i}\right)}, \prod_{i=1}^{n} a_{i 2}^{\tau\left(\alpha_{i}\right)}, \prod_{i=1}^{n} a_{i 3}^{\tau\left(\alpha_{i}\right)}, \prod_{i=1}^{n} a_{i 4} \tau\left(\alpha_{i}\right)\right],\left(\prod_{i=1}^{n}\left(T\left(\alpha_{i}\right)\right)^{\tau\left(\alpha_{i}\right)}, 1-\prod_{i=1}^{n}\left(\left(1-I\left(\alpha_{i}\right)\right)^{\tau\left(\alpha_{i}\right)}\right),\right.\right. \\
& \left.\left.1-\prod_{i=1}^{n}\left(\left(1-F\left(\alpha_{i}\right)\right)^{\tau\left(\alpha_{i}\right)}\right)\right)\right\rangle,
\end{aligned}
$$

where $\tau\left(\alpha_{i}\right)=\frac{1+G\left(\alpha_{i}\right)}{\sum_{i=1}^{n}\left(1+G\left(\alpha_{i}\right)\right)}, G\left(\alpha_{i}\right)=\sum_{j=1, j \neq i}^{n} \operatorname{Sup}\left(\alpha_{i}, \alpha_{j}\right), \operatorname{Sup}\left(\alpha_{i}, \alpha_{j}\right)=1-\left|p\left(\alpha_{i} \succ \alpha_{j}\right)-p\left(\alpha_{j} \succ \alpha_{i}\right)\right|$ is the support for $\alpha_{i}$ from $\alpha_{j}, p\left(\alpha_{i} \succ \alpha_{j}\right)$ and $p\left(\alpha_{j} \succ \alpha_{i}\right)$ are the score functions of $\alpha_{i} \succ \alpha_{j}, \alpha_{j} \succ \alpha_{i}$.

The proof of Theorem 5 can refer to Theorem 3.

Example 10. Use the data of Example 9. Then $\operatorname{SVTNPG}\left(\alpha_{1}, \alpha_{2}, \alpha_{3}\right)$ can be calculated as follows.

According to Example 9, we can get $\tau\left(\alpha_{1}\right)=0.247, \tau\left(\alpha_{2}\right)=0.248, \tau\left(\alpha_{3}\right)=0.252, \tau\left(\alpha_{4}\right)=0.253$;

$$
\text { So } \operatorname{SVTNPG}\left(\alpha_{1}, \alpha_{2}, \alpha_{3}, \alpha_{4}\right)=\langle[0.271,0.447,0.569,0.696],(0.669,0.204,0.326)\rangle \text {. }
$$

Theorem 6. Let $\alpha_{i}=\left\langle\left[a_{i 1}, a_{i 2}, a_{i 3}, a_{i 4}\right],\left(T\left(\alpha_{i}\right), I\left(\alpha_{i}\right), F\left(\alpha_{i}\right)\right)\right\rangle(i=1,2, \cdots, n)$ be a collection of positive SVTNNs. If $\operatorname{Sup}\left(\alpha_{i}, \alpha_{j}\right)=c(c \in[0,1], i \neq j, j=1,2, \cdots, n)$, then SVTNPG operator reduces to single valued trapezoidal neutrosophic geometric (SVTNG) operator as follows:

$$
\operatorname{SVTNPG}\left(\alpha_{1}, \alpha_{2}, \cdots, \alpha_{n}\right)=\operatorname{SVTNG}\left(\alpha_{1}, \alpha_{2}, \cdots, \alpha_{n}\right)=\oplus_{i=1}^{n}\left(\alpha_{i}^{1 / n}\right)
$$

The proof of Theorem 6 can refer to Theorem 4 .

\section{A MCGDM Method Based on Possibility Degree and Power Aggregation Operators under Single Valued Trapezoidal Neutrosophic Environment}

In this section, the possibility degrees of SVTNNs, single trapezoidal neutrosophic power weighted aggregation operators are applied to MCGDM problems single valued trapezoidal neutrosophic information.

For a MCGDM problems with single valued trapezoidal neutrosophic information, assume that the set of alternatives is $B=\left\{B_{1}, B_{2}, \cdots, B_{m}\right\}, D=\left\{D_{1}, D_{2}, \cdots, D_{t}\right\}$ is the set of decision-makers who evaluate the alternatives according to the criteria $C=\left\{C_{1}, C_{2}, \cdots, C_{n}\right\}$. The evaluation information $\alpha_{i j}^{y}(i=1,2, \cdots, m ; j=1,2, \cdots, n ; y=1,2, \cdots, t)$ which is described by positive SVTNNs, can be given by decision-makers $D_{y}(y=1,2, \cdots, t)$ when they assess the alternatives $B_{i}(i=1,2, \cdots, m)$ with respect to the criteria $C_{j}(j=1,2, \cdots, n)$ and then the decision matrices $R_{y}=\left(\alpha_{i j}^{y}\right)_{m \times n}$ are obtained. A method of determining the ranking of the alternatives is introduced here and the decision-making procedures are shown as follows.

Step 1. Normalize the decision matrices.

Normalize the decision-making information $\alpha_{i j}^{y}$ in the matrices $R_{y}=\left(\alpha_{i j}^{y}\right)_{m \times n}$. The criteria can be classified into the benefit type and the cost type. For the benefit-type criterion, the form of the evaluation information needs no change; but for the cost-type criterion, the negation operator is used.

The normalization of the decision matrices can be represented as follows:

$$
\left\{\begin{array}{ll}
\widetilde{\alpha}_{i j}^{y}=\alpha_{i j}^{y} & , C_{j} \in B_{T} \\
\tilde{\alpha}_{i j}^{y}=n e g\left(\alpha_{i j}^{y}\right) & , C_{j} \in C_{T}
\end{array},\right.
$$

where $B_{T}$ is the set of benefit-type criteria and $C_{T}$ is the set of cost-type criteria. 
The normalized decision matrices are denoted as $\bar{R}_{y}=\left(\widetilde{\alpha}_{i j}^{y}\right)_{m \times n}$.

Step 2. Aggregate the values of alternatives on each criterion to get the collective SVTNNs.

Based on the Definitions 12 or 13, the collective SVTNNs $\alpha_{i j}$ or $\widetilde{\alpha}_{i j}$ can be gotten by SVTNPA or SVTNPG operator, the aggregation values of decision-makers on each alternative are as follows:

$$
\alpha_{i y}=\operatorname{SVTNPA}\left(\widetilde{\alpha}_{i 1}^{y}, \widetilde{\alpha}_{i 2}^{y}, \cdots, \widetilde{\alpha}_{i n}^{y}\right) \text { or } \widetilde{\alpha}_{i y}=\operatorname{SVTNPG}\left(\widetilde{\alpha}_{i 1}^{y}, \widetilde{\alpha}_{i 2}^{y}, \cdots, \widetilde{\alpha}_{i n}^{y}\right) .
$$

Then the collective preference matrix $P=\left(\alpha_{i y}\right)_{m \times y}$ or $\widetilde{P}=\left(\widetilde{\alpha}_{i y}\right)_{m \times y}$ can be obtained.

Step 3. Aggregate the values of alternative on each decision-maker to get the overall SVTNNs.

Based on the Definitions 12 or 13, the overall SVTNNs $\alpha_{i j}$ or $\widetilde{\alpha}_{i j}$ can be gotten by SVTNPA or SVTNPG operator, the aggregation values of alternative on each decision-maker are as follows:

$$
\beta_{i}=\operatorname{SVTNPA}\left(\alpha_{i 1}, \alpha_{i 2}, \cdots, \alpha_{i t}\right) \text { or } \widetilde{\beta}_{i}=\operatorname{SVTNPA}\left(\widetilde{\alpha}_{i 1}, \widetilde{\alpha}_{i 2}, \cdots, \widetilde{\alpha}_{i t}\right) .
$$

Then the coverall preference matrix $K=\left(\beta_{i}\right)$ or $\widetilde{K}=\left(\widetilde{\beta}_{i}\right)$ can be obtained.

Step 4. Calculate the possibility degrees of the assessment values of each alternative superior than other alternatives' values.

Based on Definition 9, the possibility degrees of $\beta_{i} \succ \beta_{i^{\prime}}\left(i \neq i^{\prime}\right)$ or $\widetilde{\beta}_{i} \succ \widetilde{\beta}_{i^{\prime}}\left(i \neq i^{\prime}\right)$ can be obtained. The matrix of $p\left(\beta_{i} \succ \beta_{i^{\prime}}\right)$ or $p\left(\widetilde{\beta}_{i} \succ \widetilde{\beta}_{i^{\prime}}\right)$ can be represented as $U=\left(p\left(\beta_{i} \succ \beta_{i^{\prime}}\right)\right)_{m \times m}$ or $\widetilde{U}=\left(p\left(\widetilde{\beta}_{i} \succ \widetilde{\beta}_{i^{\prime}}\right)\right)_{m \times m}$.

Step 5. Calculate the collective possibility degree index of each alternative to derive the overall values of the alternatives.

Aggregate $U$ or $\widetilde{U}$ to get the overall possibility degree index $p\left(B_{i}\right)$ of the alternative $B_{i}$ by using the following functions:

$$
p\left(B_{i}\right)=\frac{1}{m-1} \sum_{i^{\prime}=1, i^{\prime} \neq i}^{m} p\left(\beta_{i} \succ \beta_{i^{\prime}}\right) \text { or } \widetilde{p}\left(B_{i}\right)=\frac{1}{m-1} \sum_{i^{\prime}=1, i^{\prime} \neq i}^{m} p\left(\widetilde{\beta}_{i} \succ \widetilde{\beta}_{i^{\prime}}\right) .
$$

Then the overall possibility degree index matrix $Q=\left(p\left(B_{i}\right)\right)^{T}$ or $\widetilde{Q}=\left(\widetilde{p}\left(B_{i}\right)\right)^{T}$ can be obtained.

Step 6. Rank the alternatives and select the best one.

According to the results obtained in Step 5, rank the alternatives by the overall values in descending order and the first order alternative is the best.

\section{Illustrative Example}

In this section, a green supplier selection problem is used to illustrate the validity and effectiveness of the developed method.

\subsection{Background}

The following case background is adapted from [51].

In recent years, more and more people pay attention the serious environmental problems caused badly by the rapid economic development of all over the world. The green supply chain management becomes imperative under this situation because of its advantages on the sustainable development of economics and protection of environment. Meanwhile, it can bring tremendous economic benefit and competitive strengthen for the enterprises. 
Motivated by the advantages of green supply chain management, Shanghai General Motors (SGM) Company wants to select the most appropriate green supplier as its cooperative alliance. After pre-evaluation, four suppliers become the final alternatives for further evaluation, including Howden Hua Engineering Company $\left(B_{1}\right)$, Sino Trunk $\left(B_{2}\right)$, Taikai Electric Group Company $\left(B_{3}\right)$ and Shantui construction machinery Company $\left(B_{4}\right)$. SGM employs four experts $\left(D_{y}(y=1,2,3,4)\right)$ coming from the departments of production, purchasing, quality inspection, engineering to form a group of decision-makers for evaluating the four suppliers $B_{i}(i=1,2,3,4)$ according the product quality $\left(C_{1}\right)$, technology capability $\left(C_{2}\right)$, pollution control $\left(C_{3}\right)$ and environment management $\left(C_{4}\right)$. The four experts $D_{y}(y=1,2,3,4)$ give their assessment information about the four green suppliers $B_{i}(i=1,2,3,4)$ according to the four criteria $\left(C_{j}(j=1,2,3,4)\right)$. Assume that the four experts' attitudes on evaluating the four green suppliers are neutral, that is $\lambda=0.5$. The assessment information $\alpha_{i j}^{y}(i=1,2,3,4 ; j=1,2,3,4 ; y=1,2,3,4)$ is described by SVTNNs and the decision matrices are shown in $R_{1}, R_{2}, R_{3}$ and $R_{4}$.

$$
\begin{aligned}
& c_{1}
\end{aligned}
$$

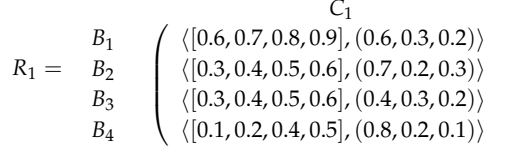

$$
\begin{aligned}
& \mathrm{C}_{2} \\
& \langle[0.3,0.4,0.5,0.6],(0.6,0.2,0.4)\rangle \\
& \langle[0.4,0.5,0.6,0.7],(0.5,0.2,0.3)\rangle \\
& \langle[0.3,0.4,0.5,0.7],(0.6,0.1,0.3)\rangle \\
& \langle[0.3,0.4,0.6,0.7],(0.2,0.5,0.4)\rangle
\end{aligned}
$$
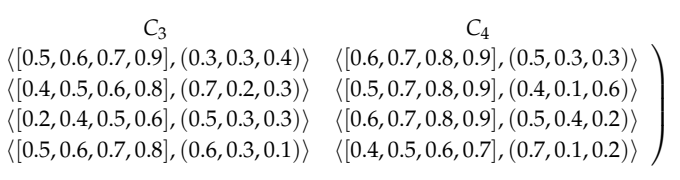

\subsection{The Procedures of Single Valued Trapezoidal Neutrosophic MCGDM Method}

The proposed MCGDM method is used for determining the ranking of the green suppliers.

Step 1. Normalize the decision matrices.

The four criteria $C_{j}(j=1,2,3,4)$ are regarded as the benefit-type criterion, so the decision matrices change nothing.

Step 2. Aggregate the values of the four alternatives on each criterion to get the collective SVTNNs.

Use the SVTNPA or SVTNPG operator to aggregate the values of four alternatives on each criterion, the collective SVTNNs are obtained shown in $P$ and $\widetilde{P}$.

$D_{1}$ $P=\begin{gathered}B_{1} \\ B_{2} \\ B_{3} \\ B_{4}\end{gathered}\left(\begin{array}{c}\langle[0.50,0.60,0.70,0.82],(0.49,0.28,0.32)\rangle \\ \langle[0.40,0.53,0.63,0.75],(0.55,0.17,0.40)\rangle \\ \langle[0.32,0.42,0.57,0.67],(0.57,0.27,0.20)\rangle\end{array}\right.$ $D_{1}$ $\left.\widetilde{P}=\begin{array}{l}B_{1} \\ B_{2} \\ B_{3} \\ B_{4}\end{array}\right\}$ $\langle[0.48,0.58,0.69,0.81],(0.48,0.28,0.33)\rangle$
$\langle[0.39,0.51,0.62,0.74],(0.56,0.18,0.39)\rangle$
$\langle[0.32,0.46,0.56,0.69],(0.49,0.28,0.25)\rangle$
$\langle[0.27,0.39,0.56,0.66],(0.52,0.29,0.21)\rangle$ $\langle[0.12,0.25,0.39,0.59],(0.57,0.24,0.25)\rangle\langle[0.14,0.28,0.42,0.57],(0.49,0.34,0.20)\rangle$ $\langle[0.40,0.58,0.68,0.83],(0.53,0.32,0.25)\rangle$ $\langle[0.27,0.42,0.57,0.67],(0.56,0.29,0.21)\rangle$ $\langle[0.30,0.40,0.62,0.75],(0.55,0.25,0.24)\rangle$ $\langle[0.17,0.30,0.45,0.60],(0.51,0.29,0.22)\rangle$

$$
D_{3}
$$

$D_{3}$ $\langle[0.15,0.40,0.53,0.68],(0.67,0.20,0.23)\rangle$
$\langle[0.33,0.53,0.70,0.88],(0.64,0.16,0.23)\rangle$
$\langle[0.15,0.30,0.53,0.78],(0.66,0.20,0.23)\rangle$
$\langle[0.13,0.25,0.40,0.60],(0.58,0.22,0.25)\rangle$
$D_{2}$
$\langle[0.14,0.39,0.52,0.67],(0.64,0.20,0.26)\rangle$
$\langle[0.31,0.52,0.69,0.87],(0.64,0.15,0.23)\rangle$
$\langle[0.14,0.29,0.51,0.76],(0.65,0.20,0.25)\rangle$
$\langle[0.12,0.25,0.39,0.59],(0.57,0.24,0.25)\rangle$

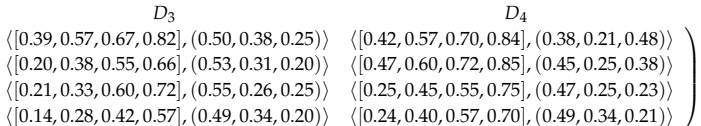

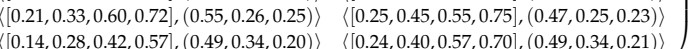

Step 3. Aggregate the values of the four alternatives on each green supplier to get the overall SVTNNs by using the SVTNPA or SVTNPG operator. 
The coverall preference matrix shown in $K$ or $\widetilde{K}$.

$$
\begin{array}{r}
K=\begin{array}{c}
B_{1} \\
B_{2} \\
B_{3}
\end{array}\left(\begin{array}{l}
{[0.36,0.54,0.65,0.79],(0.51,0.25,0.32)} \\
{[0.37,0.52,0.66,0.79],(0.55,0.22,0.31)} \\
B_{4}
\end{array}\right) \\
{[0.26,0.40,0.57,0.74],(0.54,0.25,0.23)} \\
B_{1} \\
B_{2}
\end{array}\left(\begin{array}{l}
{[0.32,0.52,0.64,0.50,0.64],(0.53,0.28,0.21)}
\end{array}\right)
$$

Step 4. Calculate the possibility degrees of the assessment values of each alternative superior than other alternatives' values to get the possibility degrees matrix $U$ or $\widetilde{U}$.

$$
\begin{array}{r}
U=\begin{array}{c}
B_{1} \\
B_{1} \\
B_{2} \\
B_{3} \\
B_{4}
\end{array}\left(\begin{array}{cccc}
- & B_{3} & B_{4} \\
0.52 & - & 0.52 & 0.54 \\
0.48 & 0.48 & - & 0.53 \\
0.47 & 0.46 & 0.47 & -
\end{array}\right) \\
B_{1} \\
\widetilde{U}=\begin{array}{cccc}
- & 0.48 & 0.51 & 0.53 \\
B_{2} \\
B_{3} \\
B_{4}
\end{array}\left(\begin{array}{cccc}
0.52 & - & 0.53 & 0.54 \\
0.49 & 0.47 & - & 0.52 \\
0.47 & 0.46 & 0.48 & -
\end{array}\right)
\end{array}
$$

Step 5. Calculate the collective possibility degree index of each alternative to derive the overall values of the alternatives.

Aggregate $U$ or $\widetilde{U}$ to get the overall possibility degree index and the overall possibility degree index matrix $Q$ or $\widetilde{Q}$.

$$
Q=\left(\begin{array}{cccc}
B_{1} & B_{2} & B_{3} & B_{4} \\
0.512 & 0.526 & 0.497 & 0.465
\end{array}\right) \quad Q=\left(\begin{array}{cccc}
B_{1} & B_{2} & B_{3} & B_{4} \\
0.510 & 0.528 & 0.494 & 0.468
\end{array}\right)
$$

Step 6. Rank the green suppliers and select the best one.

The ranking of the four green suppliers is $B_{2} \succ B_{1} \succ B_{3} \succ B_{4}$. Therefore, SGM Company will choose Sino Trunk as its cooperative alliance.

The rankings of green suppliers using the SVTNPA operators for different values of $\lambda$ are shown in Figure 1. In general, larger values of $\lambda$ are associated with relatively pessimistic decision-makers; thus, the alternatives were associated with relatively overall possibility degree index. In contrast, lower values of $\lambda$ are associated with relatively optimistic decision-makers. When the decision-makers do not indicate any preferences, the most commonly-used value $(\lambda=0.5)$ is used. 


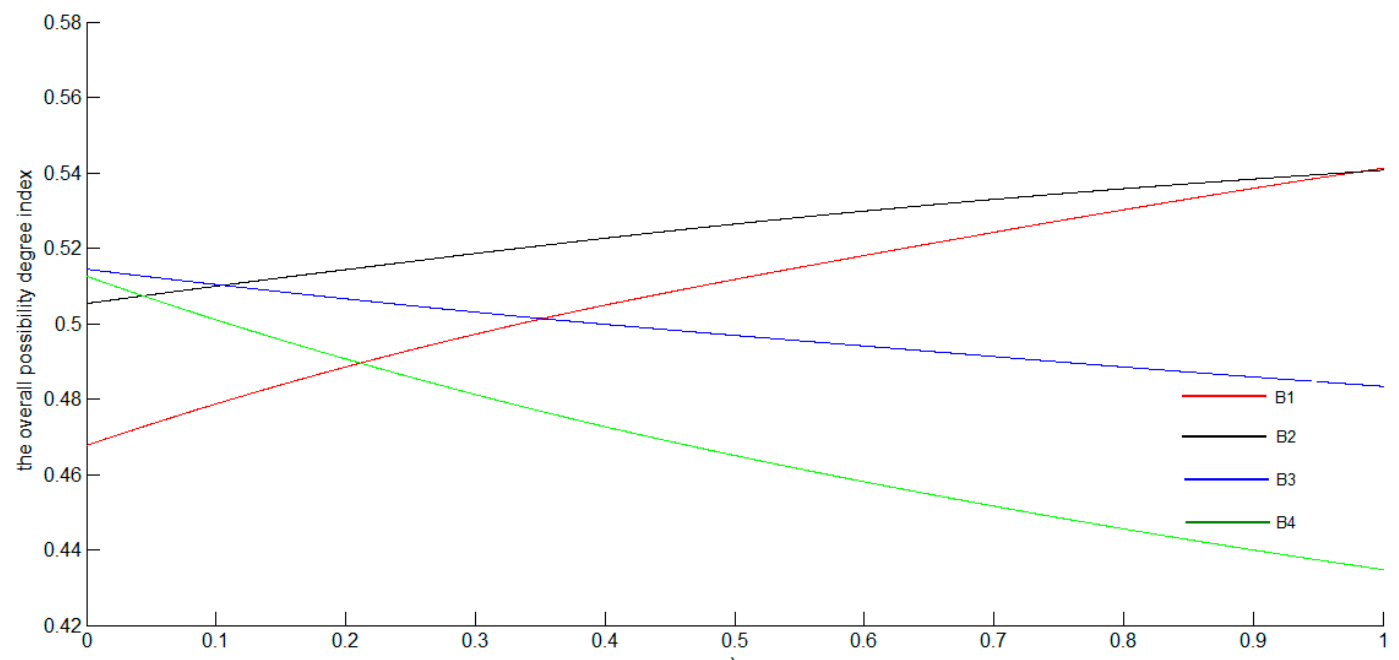

Figure 1. Rankings of various green suppliers for different values of $\lambda$.

\subsection{Comparison Analysis and Discussion}

In order to validate the accuracy of the proposed single valued trapezoidal neutrosophic MCGDM method, a comparative study is conducted based on the illustrative example in this paper and the method used for comparison was proposed by Ye [44].

When resolving the above example using the approach described in Reference [44], which involves the use of $t$ trapezoidal neutrosophic weighted arithmetic averaging (TNWAA) operator or trapezoidal neutrosophic weighted geometric averaging (TNWGA) operator with known weights to comprehensively analyze green suppliers, the weights of the decision-makers and criteria can be generated using the PA operator $\left(w_{i j}=\frac{1+G\left(\alpha_{i j}\right)}{\sum_{j=1}^{n}\left(1+G\left(\alpha_{i j}\right)\right)}, G\left(\alpha_{i j}\right)=\sum_{j=1, j \neq j^{\prime}}^{n}\left(1-\left|S\left(\alpha_{i j}\right)-S\left(\alpha_{i j^{\prime}}\right)\right|\right)\right)$ and $S\left(\alpha_{i j}\right)$ [44] is the score function value of the SVTNN $a_{i j}$. The overall values of four alternatives on each criterion obtained by using TNWAA operator are shown as the matrix $M$, the matrix $\tilde{M}$ got by using TNWGA operator.

$$
\begin{aligned}
& \begin{array}{ccccc}
D_{1} & D_{2} & D_{3} & D_{4} & D_{1} \\
B_{1} & \langle[0.52,0.62,0.72,0.85],(0.51,0.27,0.32)\rangle & \langle[0.15,0.40,0.53,0.68],(0.68,0.18,0.22)\rangle & \langle[0.40,0.57,0.67,0.82],(0.50,0.26,0.22)\rangle & \langle[0.43,0.58,0.70,0.85],(0.42,0.17,0.47)\rangle \\
B_{2} & \langle[0.40,0.53,0.63,0.75],(0.54,0.17,0.36)\rangle & \langle[0.32,0.52,0.70,0.87],(0.67,0.14,0.21)\rangle & \langle[0.27,0.42,0.57,0.67],(0.57,0.28,0.19)\rangle & \langle[0.47,0.60,0.73,0.85],(0.50,0.23,0.37)\rangle
\end{array}
\end{aligned}
$$

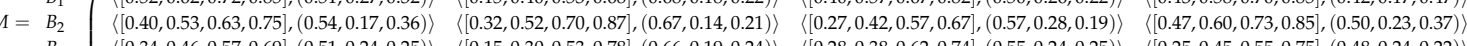

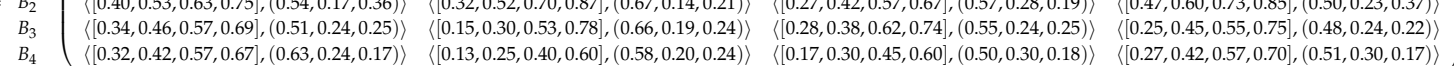

$$
\begin{aligned}
& D_{2}
\end{aligned}
$$

The collective values of the four green suppliers can also be obtained by using the TNWAA operator as the matrix $U$ or the matrix $\widetilde{U}$ by using the TNWGA operator.

$$
\begin{array}{r}
U=\begin{array}{c}
B_{1} \\
B_{2} \\
B_{3} \\
B_{4}
\end{array}\left(\begin{array}{c}
{[0.37,0.52,0.66,0.78],(0.57,0.20,0.27)} \\
\langle[0.38,0.54,0.66,0.80],(0.55,0.22,0.29)\rangle \\
\langle[0.26,0.40,0.57,0.74],(0.56,0.23,0.24)\rangle \\
\langle[0.23,0.35,0.50,0.64],(0.56,0.26,0.19)\rangle
\end{array}\right) \\
\widetilde{U}=\begin{array}{l}
B_{1} \\
B_{3} \\
B_{4}
\end{array}\left(\begin{array}{c}
\langle[0.33,0.50,0.64,0.77],(0.51,0.23,0.31)\rangle \\
\langle[0.33,0.53,0.65,0.79],(0.49,0.27,0.34)\rangle \\
\langle[0.18,0.32,0.48,0.55,0.73],(0.54,0.25,0.25)\rangle
\end{array}\right)
\end{array}
$$


Finally, the score values $s_{i}(i=1,2,3,4)$ of each green supplier can be obtained by using the score degree function show in the matrix $H$ or $\widetilde{H}$.

$$
H=\left(\begin{array}{cccc}
B_{1} & B_{2} & B_{3} & B_{4} \\
0.410 & 0.404 & 0.342 & 0.301
\end{array}\right) \quad \widetilde{H}=\left(\begin{array}{llll}
B_{1} & B_{2} & B_{3} & B_{4} \\
0.371 & 0.362 & 0.317 & 0.267
\end{array}\right)
$$

So, the ranking is $B_{1} \succ B_{2} \succ B_{3} \succ B_{4}$ and the best green supplier obtained by using the approach in Reference [44] is $B_{1}$. The ranking results of different methods can be shown in Table 1 .

Table 1. The ranking results of different methods.

\begin{tabular}{lll}
\hline Methods & Operators & Ranking of Alternatives \\
\hline \multirow{2}{*}{ The method in Reference [44] } & NNTWA operator & $B_{1} \succ B_{2} \succ B_{4} \succ B_{3}$ \\
& NNTWG operator & $B_{4} \succ B_{2} \succ B_{1} \succ B_{3}$ \\
\hline \multirow{2}{*}{ The proposed method } & SVTNPA operator and the & $B_{2} \succ B_{1} \succ B_{3} \succ B_{4}$ \\
& $\begin{array}{l}\text { possibility degrees SVTNNs } \\
\text { SVTNPG operator and the } \\
\text { possibility degrees SVTNNs }\end{array}$ & $B_{2} \succ B_{1} \succ B_{4} \succ B_{3}$ \\
\hline
\end{tabular}

From Table 1, it can be seen results of the ranking on the four green suppliers obtained by the proposed single trapezoidal neutrosophic MCGDM method in this paper is quite different from that the ranking obtained by the method introduced in Reference [44]. The main reasons are summarized as follows.

(a) The new operations of SVTNNs defined in this paper, which take the conservative and reliable principle, can take account of the correlation between trapezoidal fuzzy numbers and three membership degrees of SVTNNs. However, the operations in Reference [44] divide the trapezoidal fuzzy numbers and three membership degrees of SVTNNs into two parts and calculate them separately, which make aggregating results deviate from the reality.

(b) The new comparison of SVTNNs proposed in this paper has some crucial advantages over comparison of SVTNNs based on the score degree function in Reference [44], which can take the preference of decision-makers into consideration.

(c) The relationship among the aggregation information, which exists in the aggregation process of in practical MCDM problems, is ignored [44]. Whereas, the SVTNPA and SVTNPG operators, which can effectively take the relationship among the assessment information being aggregated into consideration and in this paper, the advantages of the possibility degree of SVTNNs are combined to rank the uncertain information reasonably and accurately from the probability viewpoint. Hence, the ranking result of this paper is more objective and reasonable than that obtained by using the operators in Reference [44].

\section{Conclusions}

In order to improve the reasonability and effectiveness of the methods on dealing with single valued trapezoidal neutrosophic MCGDM problems, also overcome the limitations of the existing approaches. In this paper, a single valued trapezoidal neutrosophic MCGDM method is proposed form the possibility degree of SVTNNs and the single valued trapezoidal neutrosophic power aggregation operators. Firstly, the new operations of SVTNNs are proposed for avoiding information loss and distortion, the possibility degrees of SVTNNs are proposed from the probability viewpoint. Based on the proposed operations and possibility degrees, SVTNPA and SVTNPG operators are proposed. Furthermore, a single valued trapezoidal neutrosophic MCGDM method based on SVTNPA, SVTNPG operator and the possibility degrees of SVTNNs is developed. The prominent advantages of the proposed method are not only its ability to effectively deal with the preference information 
expressed by SVTNNs but also the consideration of the relationship among the information being aggregated in the process on dealing with the practical MCGDM problems and the advantage of the possibility degrees of SVTNNs, which can avoid information loss and distortion, is combined. Thus, the final results are more scientific and reasonable. Finally, the method is applied to a practical problem on selecting the most appropriate green supplier for SGM Company, meanwhile, the comparison with other method is carried on and demonstrates its feasibility and effectiveness in dealing with MCGDM problems.

In future research, the developed method will be extended to other domains, such as personnel selection and medical diagnosis.

Author Contributions: X.W. proposed the revised operation rules, J.Q. gave the MDGDM approaches and SVTNPA, SVTNPG operator, all the author wrote the manuscript and revised the final version.

Funding: This work is supported by the National Natural Science Foundation of China (Grant No. 7170011164, 71571193), the Natural Science Foundation of Hubei Province under Grant No. (2016CFB519) and the Project of Youth and Middle-aged Innovation Team of Science and Technology of Hubei Province Grant No. (T201711).

Conflicts of Interest: The authors declare that there is no conflict of interests regarding the publication of this paper.

\section{References}

1. Zadeh, L.A. Fuzzy sets. Inf. Control 1965, 8, 338-353. [CrossRef]

2. Atanassov, K.T. Intuitionistic fuzzy sets. Fuzzy Sets Syst. 1986, 20, 87-96. [CrossRef]

3. Torra, V.; Narukawa, Y. On hesitant fuzzy sets and decision. In Proceedings of the 18th IEEE International Conference on Fuzzy Systems in Fuzzy Systems, Jeju Island, Korea, 20-24 August 2009; pp. 1378-1382.

4. Medina, J.; Ojeda-Aciego, M. Multi-adjoint t-concept lattices. Inf. Sci. 2010, 180, 712-725. [CrossRef]

5. Pozna, C.; Minculete, N.; Precup, R.-E.; Kóczy, L.T.; Ballagi, A. Signatures: Definitions, operators and applications to fuzzy modelling. Fuzzy Sets Syst. 2012, 201, 86-104. [CrossRef]

6. Nowaková, J.; Prílepok, M.; Snášel, V. Medical image retrieval using vector quantization and fuzzy s-tree. J. Med. Syst. 2017, 41, 18. [CrossRef] [PubMed]

7. Kumar, A.; Kumar, D.; Jarial, S.K. A hybrid clustering method based on improved artificial bee colony and fuzzy c-means algorithm. Int. J. Artif. Intell. 2017, 15, 40-60.

8. Smarandache, F. A Unifying Field in Logics: Neutrosophic Logic, 4th ed. Available online: https://www.researchgate.net/publication/2098003_A_Unifying_Field_in_Logics_Neutrosophic_Logic_ Neutrosophic_Set_Neutrosophic_Probability_and_Statistics_fourth_edition (accessed on 26 July 2018).

9. Smarandache, F. Neutrosophic set, a generalisation of the intuitionistic fuzzy sets. Int. J. Pure Appl. Math. 2005, 24, 287-297.

10. Wu, X.H.; Wang, J.Q.; Peng, J.J.; Qian, J. A novel group decision-making method with probability hesitant Interval neutrosphic set and its application in middle level manager's selection. Int. J. Uncertain. Quantif. 2018, 8, 291-319. [CrossRef]

11. Wang, H.B.; Smarandache, F.; Zhang, Y.; Sunderraman, R. Single Valued Neutrosophic Sets. Available online: https:/ / www.researchgate.net/publication/262034561_Single_Valued_Neutrosophic_Sets (accessed on 28 July 2018).

12. Wang, H.B.; Smarandache, F.; Sunderraman, R.; Zhang, Y.Q. Interval Neutrosophic Sets and Logic: Theory and Applications in Computing; Hexis: Phoenix, AZ, USA, 2005.

13. Peng, J.J.; Wang, J.Q.; Wu, X.H.; Wang, J.; Chen, X.H. Multi-valued neutrosophic sets and power aggregation operators with their applications in multi-criteria group decision-making problems. Int. J. Comput. Intell. Syst. 2015, 8, 345-363. [CrossRef]

14. Pramanik, S.; Mondal, K. Cosine similarity measure of rough neutrosophic sets and its application in medical diagnosis. Glob. J. Adv. Res. 2015, 2, 212-220.

15. Ye, S.; Fu, J.; Ye, J. Medical diagnosis using distance-based similarity measures of single valued neutrosophic multisets. Neutrosophic Sets Syst. 2015, 7, 47-52. 
16. Ma, Y.X.; Wang, J.Q.; Wang, J.; Wu, X.H. An interval neutrosophic linguistic multi-criteria group decision-making method and its application in selecting medical treatment options. Neural Comput. Appl. 2017, 28, 2745-2765. [CrossRef]

17. Kraipeerapun, P.; Fung, C.C. Binary classification using ensemble neural networks and interval neutrosophic sets. Neurocomputing 2009, 72, 2845-2856. [CrossRef]

18. Liang, R.X.; Wang, J.Q.; Zhang, H.Y. A multi-criteria decision-making method based on single-valued trapezoidal neutrosophic preference relations with complete weight information. Neural Comput. Appl. 2017. [CrossRef]

19. Zhang, Z.M.; Wu, C. A novel method for single-valued neutrosophic multi-criteria decision making with incomplete weight information. Neutrosophic Sets Syst. 2014, 4, 35-49.

20. Tian, Z.P.; Wang, J.; Wang, J.Q.; Zhang, H.Y. Simplified neutrosophic linguistic multi-criteria group decision-making approach to green product development. Group Decis. Negot. 2017, 26, 597-627. [CrossRef]

21. Liu, P.D.; Wang, Y.M. Multiple attribute decision-making method based on single-valued neutrosophic normalized weighted Bonferroni mean. Neural Comput. Appl. 2014, 25, 2001-2010. [CrossRef]

22. Liu, P.D.; Chu, Y.C.; Li, Y.W.; Chen, Y.B. Some generalized neutrosophic number Hamacher aggregation operators and their application to group decision making. Int. J. Fuzzy Syst. 2014, 16, 242-255.

23. Şahin, R. Multi-Criteria Neutrosophic Decision Making Method Based on Score and Accuracy Functions under Neutrosophic Environment. Available online: https:/ / www.researchgate.net/publication/269722070_ Multi-criteria_neu-trosophic_decision_making_method_based_on_score_and_accuracy_functions_under_ neutrosophic_environment (accessed on 29 July 2018).

24. Sun, H.X.; Yang, H.X.; Wu, J.Z.; Ouyang, Y. Interval neutrosophic numbers Choquet integral operator for multi-criteria decision making. J. Intell. Fuzzy Syst. 2015. [CrossRef]

25. Ye, J. Multiple attribute decision-making method based on the possibility degree ranking method and ordered weighted aggregation operators of interval neutrosophic numbers. J. Int. Fuzzy Syst. 2015, 28, 1307-1317.

26. Zhang, H.Y.; Wang, J.Q.; Chen, X.H. Interval neutrosophic sets and their application in multicriteria decision making problems. Sci. World J. 2014. [CrossRef] [PubMed]

27. Şahin, R.; Küçük, A. Subsethood measure for single valued neutrosophic sets. J. Intell. Fuzzy Syst. 2014. [CrossRef]

28. Ye, J. Multicriteria decision-making method using the correlation coefficient under single-valued neutrosophic environment. Int. J. Gen. Syst. 2013, 42, 386-394. [CrossRef]

29. Ye, J. Single valued neutrosophic cross-entropy for multicriteria decision making problems. Appl. Math. Model. 2014, 38, 1170-1175. [CrossRef]

30. Ye, J. Multiple attribute group decision-making method with completely unknown weights based on similarity measures under single valued neutrosophic environment. J. Intell. Fuzzy Syst. 2014. [CrossRef]

31. Wu, X.H.; Wang, J.Q.; Peng, J.J.; Chen, X.H. Cross-entropy and prioritized aggregation operator with simplified neutrosophic sets and their application in multi-criteria decision-making problems. Int. J. Fuzzy Syst. 2016, 18, 1104-1116. [CrossRef]

32. Broumi, S.; Smarandache, F. Correlation coefficient of interval neutrosophic set. Appl. Mechan. Mater. 2014, 436, 511-517. [CrossRef]

33. Broumi, S.; Smarandache, F. Cosine similarity measure of interval valued neutrosophic sets. Neutrosophic Sets Syst. 2014, 5, 15-20.

34. Broumi, S.; Smarandache, F. New Distance and Similarity Measures of Interval Neutrosophic Sets. Available online: http:/ / fs.unm.edu/NewDistanceAndSimilarity.pdf (accessed on 29 July 2018).

35. Ye, J. Similarity measures between interval neutrosophic sets and their applications in multicriteria decision-making. J. Intell. Fuzzy Syst. 2014, 26, 165-172.

36. Şahin, R.; Karabacak, M. A multi attribute decision making method based on inclusion measure for interval neutrosophic sets. Int. J. Eng. Appl. Sci. 2015, 2, 13-15.

37. Biswas, P.; Pramanik, S.; Giri, B.C. Entropy based grey relational analysis method for multi-attribute decision making under single valued neutrosophic assessments. Neutrosophic Sets Syst. 2014, 2, 102-110.

38. Peng, J.J.; Wang, J.Q.; Zhang, H.Y.; Chen, X.H. An outranking approach for multi-criteria decision-making problems with simplified neutrosophic sets. Appl. Soft Comput. 2014, 25, 336-346. [CrossRef] 
39. Şahin, R.; Yiğider, M. A Multi-criteria neutrosophic group decision making metod based TOPSIS for supplier selection. Appl. Math. Inf. Sci. 2014, 10, 1843-1852. [CrossRef]

40. Chi, P.P.; Liu, P.D. An extended TOPSIS method for the multiple attribute decision making problems based on interval neutrosophic set. Neutrosophic Sets Syst. 2013, 1, 63-70.

41. Wang, J.Q.; Li, X.E. TODIM Method with Multi-Valued Neutrosophic Sets. Control and Decision. Available online: http:/ / www.cnki.net/kcms/detail/21.1124.TP.20150108.1400.005.html (accessed on 30 July 2018).

42. Wu, X.H.; Wang, J.Q. Cross-entropy measures of multi-valued neutrosophic sets and its application in selecting middle-level manager. Int. J. Uncertain. Quantif. 2017, 7, 155-176. [CrossRef]

43. Wang, J.Q.; Zhang, Z. Aggregation operators on intuitionistic trapezoidal fuzzy number and its application to multi-criteria decision making problems. J. Syst. Eng. Electron. 2009, 20, 321-326.

44. Ye, J. Some weighted aggregation operators of trapezoidal neutrosophic numbers and their multiple attribute decision making method. Available online: http://www.gallup.unm.edu/ smarandache/ SomeWeightedAggregationOperators.pdf (accessed on 30 July 2018).

45. Yager, R.R. The power average operator. IEEE Trans. Syst. Man Cybern. Part A Syst. Hum. 2001, 31, 724-731. [CrossRef]

46. $\mathrm{Xu}$, Z.S.; Yager, R.R. Power-geometric operators and their use in group decision making. IEEE Trans. Fuzzy Syst. 2010, 18, 94-105.

47. Dubois, D.; Prade, H. Ranking fuzzy numbers in the setting of possibility theory. Inf. Sci. 1983, 30, $183-224$. [CrossRef]

48. Wang, J.Q.; Nie, R.R.; Zhang, H.Y.; Chen, X.H. New operators on triangular intuitionistic fuzzy numbers and their applications in system fault analysis. Inf. Sci. 2013, 251, 79-95. [CrossRef]

49. Nakahara, Y.; Sasaki, M.; Gen, M. On the linear programming problems with interval coefficients. Comput. Ind. Eng. 1992, 23, 301-304. [CrossRef]

50. Wan, S.P.; Dong, J.Y. A possibility degree method for interval-valued intuitionistic fuzzy multi-attribute group decision making. J. Comput. Syst. Sci. 2014, 80, 237-256. [CrossRef]

51. Wan, S.P.; Dong, J.Y. Power geometric operators of trapezoidal intuitionistic fuzzy mumbers and application to multi-attribute group decision making. Appl. Soft Comput. 2015, 29, 153-168. [CrossRef]

(C) 2018 by the authors. Licensee MDPI, Basel, Switzerland. This article is an open access article distributed under the terms and conditions of the Creative Commons Attribution (CC BY) license (http://creativecommons.org/licenses/by/4.0/). 